\title{
Influence of injection parameters on fueling efficiency of supersonic molecular beam
} injection into turbulent fusion plasmas

Avdeeva, G.; Naulin, V.; Nielsen, A. H.; Juul Rasmussen, J.; Thrysøe, A.S.

Published in:

Physics of Plasmas

Link to article, DOI:

$10.1063 / 5.0002858$

Publication date:

2020

Document Version

Publisher's PDF, also known as Version of record

Link back to DTU Orbit

Citation (APA):

Avdeeva, G., Naulin, V., Nielsen, A. H., Juul Rasmussen, J., \& Thrysøe, A. S. (2020). Influence of injection parameters on fueling efficiency of supersonic molecular beam injection into turbulent fusion plasmas. Physics of Plasmas, 27(6), [062515]. https://doi.org/10.1063/5.0002858

\section{General rights}

Copyright and moral rights for the publications made accessible in the public portal are retained by the authors and/or other copyright owners and it is a condition of accessing publications that users recognise and abide by the legal requirements associated with these rights.

- Users may download and print one copy of any publication from the public portal for the purpose of private study or research.

- You may not further distribute the material or use it for any profit-making activity or commercial gain

- You may freely distribute the URL identifying the publication in the public portal 


\section{Influence of injection parameters on fueling efficiency of supersonic molecular beam injection into turbulent fusion plasmas}

Cite as: Phys. Plasmas 27, 062515 (2020); https://doi.org/10.1063/5.0002858

Submitted: 28 January 2020 . Accepted: 09 June 2020 . Published Online: 26 June 2020

(D) G. Avdeeva, (D) V. Naulin, (D) A. H. Nielsen, (D) J. Juul Rasmussen, and (iD A. S. Thrysøe

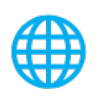

\section{ARTICLES YOU MAY BE INTERESTED IN}

Role of sheared $\mathrm{E} \times \mathrm{B}$ flow in self-organized, improved confinement states in magnetized plasmas

Physics of Plasmas 27, 060501 (2020); https://doi.org/10.1063/1.5142734

Examination of synthetic gas puff imaging diagnostic data from a gyrokinetic turbulence code Physics of Plasmas 27, 062512 (2020); https://doi.org/10.1063/5.0002876

Experimental determination of the thermal, turbulent, and rotational ion motion and magnetic field profiles in imploding plasmas

Physics of Plasmas 27, 060901 (2020); https://doi.org/10.1063/5.0009432

\section{AIP Advances Fluids and Plasmas Collection}




\title{
Influence of injection parameters on fueling efficiency of supersonic molecular beam injection into turbulent fusion plasmas
}

Cite as: Phys. Plasmas 27, 062515 (2020); doi: 10.1063/5.0002858

Submitted: 28 January 2020 . Accepted: 9 June 2020 .

Published Online: 26 June 2020

G. Avdeeva, (D) V. Naulin, a) (D) A. H. Nielsen, (D) J. Juul Rasmussen, (D) and A. S. Thrysøe (D)

\author{
AFFILIATIONS \\ PPFE, Department of Physics, DTU, DK-2800 Kgs. Lyngby, Denmark \\ a) Author to whom correspondence should be addressed: vona@fysik.dtu.dk
}

\begin{abstract}
The fueling efficiency of supersonic molecular beam injection (SMBI) depends on injection parameters. In this article, we report on simulations of SMBI into turbulent edge fusion plasmas. Density continuity equations, which describe the dynamics of molecules and Franck-Condon atoms, are coupled with the plasma equations in the hot-edge-sol-electrostatic (HESEL) drift-fluid model. Numerical simulations, with parameters relevant to experimental conditions, are performed in order to investigate the dependency of the fueling efficiency of SMBI on the molecular beam density, velocity, and beam width. In all conditions, the efficiency grows with the increase in the molecular beam velocity. The variation in efficiency with respect to the beam density depends on the plasma pressure and the ratio of the density source, produced by ionization of neutrals, to the plasma density source provided by core fueling. The effect of the molecular beam width is pronounced only if the beam density is high and the plasma, into which the beam is injected, is relatively dense and hot. The influence of plasma fluctuations on SMBI is weak; however, a strong neutral injection leads to suppression of a turbulence transport due to relaxation of pressure gradient profiles.
\end{abstract}

Published under license by AIP Publishing. https://doi.org/10.1063/5.0002858

\section{INTRODUCTION}

Refueling of fusion plasmas during tokamak operation is essential for sustainable fusion reactions. The necessity of plasma refueling results from the radial transport, which leads to particle and energy losses across the separatrix. Refueling is also required in a burning steady state plasma where fuel is consumed and needs to be re-injected. An efficient fueling method should provide penetration of neutrals into the confined region and minimize the amount of particles ionized in the scrape-off-layer (SOL). Intensive ionization in the SOL region imposes restrictions for high throughput pumping systems, which provide the tokamak vacuum. The traditional fueling method used on fusion machines is by gas puffing (GP), which results in edge fueling and demonstrates its highest efficiency, defined as the ratio of the maximum increment of plasma density to the injected inventory, in divertor configurations with less than 20\%. ${ }^{1,2}$ Supersonic molecular beam injection (SMBI) ${ }^{3}$ is similar to GP apart from using a Laval nozzle for acceleration of molecules to supersonic speeds. SMBI has been widely used on different machines ${ }^{1,4-11}$ and demonstrated fueling efficiencies in the range of $20 \%-60 \%$. SMBI is a useful tool to perform fast plasma density control especially in H-mode where GP becomes increasingly ineffective. In addition, SMBI is characterized by lower wall retention compared to GP, essential for long pulse tokamak operations. ${ }^{1,8}$ On EAST and KSTAR, SMBI is implemented in the feedback density control system.

The efficiency of SMBI as a fueling method and its influence on the plasma depend on injection parameters and plasma conditions. A decrease in fueling efficiency with the increase in averaged plasma density before the injection was observed on Tore Supra, ${ }^{4} \mathrm{HL}-2 \mathrm{~A},{ }^{6}$ KSTAR. ${ }^{1}$ A slight rise of the fueling efficiency with the increase in the molecular beam density was demonstrated on Tore Supra. ${ }^{4}$

The absence of precise measurements of the molecular beam parameters at the entry of the plasma, low ability to vary injection parameters, and scattering of measured fueling efficiency from shot to shot complicate obtaining a comprehensive picture and quantitative description of how the efficiency of SMBI depends on various injection scenarios. Numerical simulations are a powerful tool for the investigation of fueling properties of SMBI and for determining the optimal injection parameters. Numerical simulations performed with a MHD code for supersonic gas jets ${ }^{12}$ as well as simulations performed with the trans-neut module ${ }^{13}$ pointed out that the molecular beam intensity 
is one of the main parameters determining the penetration depth of neutrals. As demonstrated in Ref. 12 only the outer layers of the high density supersonic jet are ionized, while the central part remains almost neutral and penetrates inside the plasma confined region. The decrease of the jet density leads to the fast ionization of injected neutrals in the vicinity of the separatrix. On the other hand, the ionization of the dense neutral beam results in the sharp increase in the plasma density, which terminates the beam propagation inside the plasma as shown in Ref. 14. The numerical simulations reported in Refs. 14 and 15 also demonstrate deeper penetration of neutrals with the increase in the beam velocity; the density, however, plays the most important role.

It should be noted that none of those simulations included the turbulent plasma dynamics, although experimental and theoretical works showed that the SOL region is highly intermittent. ${ }^{16-20}$ High amplitude fluctuations in the SOL region affect the molecular beam propagation by variations of ionization and dissociation rates. Selfconsistent simulations of plasma and neutrals governed by diffusion processes showed the distinct influence of the turbulence on neutral transport. ${ }^{21-25}$ In particular, those works highlighted the importance of the inclusion of plasma dynamics in the analysis of the gas puff imaging diagnostic and reduced the discrepancy in the analysis of the neutral flux across the last-closed-flux-surface (LCFS) in the turbulent simulations compared to the mean field approximation. Resembling influence of the turbulence on the propagation of the supersonic molecular beam is expected; however, this effect should be smaller for SMBI than for GP, as the mean free part of the molecular beam governed by the convection transport is longer compared to the diffusive neutrals and large compared to the turbulence correlation length. In addition, the inclusion of the plasma dynamics in the simulations of SMBI is also important to imitate the fast energy and plasma particle transport across the LCFS, which is found to be much faster than the neoclassical diffusion transport. Typical time of the plasma fluctuations is microsecond unlike the millisecond time scale, which was used in simulations before. ${ }^{4,9,14}$

This work presents analysis of the fueling efficiency of SMBI based on combined fluid simulations of supersonic molecular beam and plasma turbulence. The plasma interacts with the beam through dissociation and ionization of molecules by electron impact. The dynamics of the Franck-Condon atoms resulting from dissociation of molecules are included in the model. This model is used to investigate how the fueling efficiency scales with the molecular beam parameters, namely the intensity, velocity, and width of the beam in different plasma conditions. This work also presents analysis of the influence of turbulence on SMBI fueling and vice versa, i.e., the influence of neutral injection on plasma dynamics in the edge/SOL regions. The results are discussed in comparison with findings from previous numerical models applied for the investigation of SMBI.

This paper is organized as follows: The numerical model is described in Sec. II. The simulation setup and initial parameters are discussed in Sec. III. The scaling of the efficiency as a function of the beam parameters is presented in Sec. IV. Section IV D is devoted to the analysis of turbulence, in particular, its influence on the molecular beam propagation and suppression of the turbulent transport caused by SMBI. The comparison of results with findings from previous numerical models is presented in Sec. V. The results are summarized in Sec. VI.

\section{MODEL}

The neutral beam injected into the plasma undergoes molecular and atomic reactions by interactions with ions and electrons. For simplification, the excited states of molecules and atoms are not considered, as it would dramatically increase the computation time. This simplification allows for performing long simulations with sufficient data for statistical analysis. The dissociation and ionization of molecules by electron impact have the biggest reaction rates among the various processes between hydrogen isotopes and plasma electrons. ${ }^{26}$ Although the ionization of molecules is a two step process through the existence of $\mathrm{H}_{2}^{+}$, the assumption of an instantaneous dissociation of $\mathrm{H}_{2}^{+}$after its creation is applied in the model. This assumption is based on the higher cross section of the dissociation of $\mathrm{H}_{2}^{+}$compared to other considered reactions. The deuterium neutral injection is considered in the model and we assume similar reaction rate coefficients for hydrogen and deuterium. The dissociation of molecules results in creation of Franck-Condon atoms with the same direct velocity as injected molecules and with a thermal energy of $2 \mathrm{eV}$. These atoms are included in the model as a separate neutral species. Atoms are subjected to electron impact ionization.

Electron neutral interactions result in sink and source terms, which are added to the equations for neutrals and plasma. Neutral-neutral interactions are omitted since their cross section is small compared to the dissociation and ionization processes.

To perform self-consistent plasma-supersonic molecular beam simulations, the continuity equations for the molecules and atoms are coupled with a drift-fluid model describing magnetized plasma.

The HESEL (hot-edge-sol-electrostatic) model ${ }^{27,28}$ describes the dynamics of the plasma density, generalized vorticity, and ion and electron pressure at the outboard mid-plane of a tokamak in a 2D slab geometry perpendicular to a magnetic field. The HESEL model has successfully reproduced the statistical properties and profiles of plasmas in EAST, ${ }^{17}$ MAST, ${ }^{18}$ and TCV. ${ }^{19}$

The HESEL model is a first principles physical model; however, some assumptions are applied mainly for computational expediency.

First, this model is a reduced drift-fluid model; therefore, all assumptions implied during the drift-ordering procedure are applicable to the HESEL model. More detailed information about the driftordering procedure can be found in Refs. 27 and 29.

The HESEL model implies the assumption of plasma quasineutrality.

Another simplifications concern reduction of the model to two spatial dimensions and parametrization in the parallel direction, i.e., direction parallel to the magnetic field. The HESEL model is a $2 \mathrm{D}$ model and describes the dynamics of low-frequency turbulence in the region encompassing $30^{\circ}$ above and below the outboard mid-plane (see also Ref. 28 and the reference there). Therefore, the model uses the assumption of a finite ballooning length for the parallel transport, equal to $L_{b}=q R$. The equations are solved in slab geometry with the unit vector $z$ along the inhomogeneous toroidal magnetic field. The magnetic field is approximated by $B(r)=B_{0} /\left(1+\frac{a}{R}+\frac{x}{R}\right)$, where $B_{0}$ is the magnetic field strength at the outboard mid-plane, $a$ and $R$ are the minor and major radii of the tokamak, and the radial coordinate $x$ is equal to zero at LCFS. The tokamak geometry and poloidal magnetic field lines are accounted for by a neo-classical Pfirsch-Schlüter correction to the diffusion coefficients 
$D_{e}=\left(1+\frac{R}{a} q_{95}^{2}\right) \frac{\rho_{e 0}^{2} \nu_{e i 0}}{\rho_{s}^{2} \Omega_{i 0}}, D_{i}=\left(1+\frac{R}{a} q_{95}^{2}\right) \frac{\rho_{i 0}^{2} \nu_{i i 0}}{\rho_{s}^{2} \Omega_{i 0}}$, where $q_{95}$ is the safety factor at $95 \%$ of the normalized plasma radius, $\rho_{e 0}, \rho_{i 0}$ are the electron and ion gyro radii, $\Omega_{i 0}$ is the characteristic ion gyro frequency, $\rho_{s}$ is the cold-ion hybrid thermal gyro-radius, and $\nu_{e i 0}$ and $\nu_{i i 0}$ are the electron-ion and ion-ion collision frequencies. In more detail, these terms are discussed in Ref. 27.

The HESEL model simulates regions with closed magnetic field lines (edge) and open magnetic field lines (SOL) with a smooth transition between them. As mentioned above, the HESEL domain covers only the small area at the outboard mid-plane of a tokamak and the divertor region is not included. An illustration of the HESEL domain is presented in Fig. 1 and the scaled picture of the HESEL domain compared to the poloidal cross section of a medium sized tokamak can be found in Ref. 30 .

At the inner part of the edge region, plasma profiles are forced to prescribed values and this acts as a source for the particles and energy, respectively. ${ }^{28}$ The forcing sources are defined through the fixed profiles $\left(n_{\text {fixed }}, p e_{\text {fixed }}, p i_{\text {fixed }}\right)$ and the relaxation time $\left(\tau_{r}\right)$. The forcing is required in the HESEL model to provide the approximation to the core region, which acts as the constant source of particles and energy in real experimental situations. Therefore, the forcing profile accounts for density sources such as NBI or other core fueling sources and provides feedback forcing of the density and pressure profiles toward given profiles at the inside of the region considered. For the plasma density $(n)$, this source is written as

$$
S_{\text {force }}=\frac{\left(n_{\text {fixed }}-n\right)}{\tau_{r}} .
$$

The difference between the edge and SOL regions is that in the open field line region parametrized parallel loses are included. The plasma propagates along the magnetic field lines with a speed of $v_{\|}=2 M_{\|} c_{s}$, where $c_{s}$ is the ion sound speed and $M_{\|}$is the parallel Mach number. ${ }^{28}$ Losses due to parallel advection and Spitzer-Härm heat electron conduction along the magnetic field lines in the SOL region are represented by the damping rates,

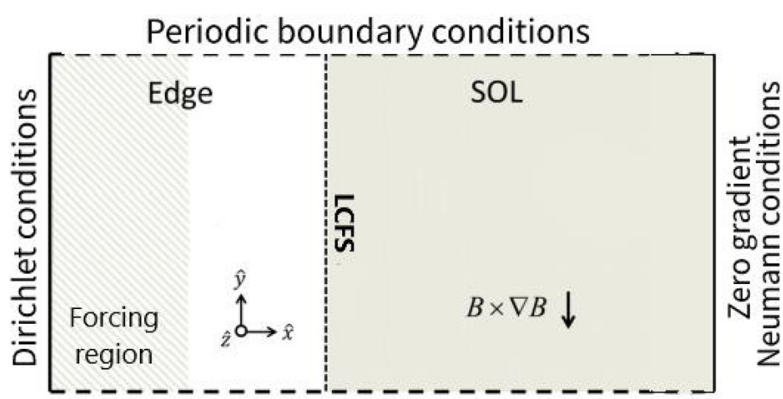

Periodic boundary conditions

FIG. 1. An illustration of the HESEL domain demonstrating the edge and SOL regions, LCFS and the coordinate system. $\mathrm{X}$ is a radial direction, $\mathrm{y}$ is equivalent to the poloidal direction. At the inner part of the edge region, i.e., forcing region, plasma profiles are forced to prescribed values. The transition between edge and $\mathrm{SOL}$ regions is set by the smooth function which mitigates particle losses across the open field lines in the SOL region.

$$
\begin{gathered}
\frac{1}{\tau_{n}}=\frac{2 M_{\|} \sqrt{\frac{T_{e}+T_{i}}{m_{i}}}}{q_{95} R}, \\
\frac{1}{\tau_{S H}}=3.16 \frac{n_{0}}{m_{e} \nu_{e i 0} T_{e 0}^{3 / 2} L_{c}^{2}},
\end{gathered}
$$

where $T_{e}$ and $T_{i}$ are the electron and ion temperatures, respectively, $m_{i}$ is the ion mass, $n_{0}$ is the reference density, $T_{e 0}$ is the electron reference temperature, and $L_{c}$ is the connection length to the divertor plates.

Particle losses in the SOL region can be written as

$$
S_{\|}=-\sigma(x) \frac{n}{\tau_{n}}
$$

where $\sigma(x)$ is the "smooth step function" which defines the transition from the edge to SOL regions in the model. ${ }^{27}$ Dirichlet boundary conditions, in agreement with the prescribed profiles, are applied at the inner radial boundary, zero gradient Neumann boundary conditions at the outer radial boundary, and periodic boundary conditions in poloidal direction (Fig. 1).

More detailed and further information (which is not directly related to and superfluous for the presented study) regarding the HESEL model and applied assumptions can be found in Refs. 27 and 28.

Sink and source terms resulting from inelastic plasma-neutral interactions are derived from the Boltzmann equation in Ref. 31 and, in particular, for the coupling with the HESEL model in Ref. 23. In order to facilitate the study of plasma-supersonic molecular beam interactions and to make the interpretation of results clearer, the reduced model presented in Ref. 23 is used for the primary investigation.

Ionization of molecules and atoms results in the density source

$$
S^{\mathrm{n}}=n\left(N_{\mathrm{mol}}\langle\sigma v\rangle_{\mathrm{mol}}^{\text {ion }}+N_{\mathrm{FC}}\langle\sigma v\rangle_{\text {atom }}^{\text {ion }}\right)=S_{\mathrm{mol}}^{\mathrm{n}}+S_{\mathrm{FC}}^{\mathrm{n}}
$$

where $n$ is the plasma density, $\langle\sigma v\rangle_{\text {mol }}^{\text {ion }}$ and $\langle\sigma v\rangle_{\text {atom }}^{\text {ion }}$ are the reaction rate coefficients for the ionization of molecules and ionization of atoms, which are approximated as functions of an electron temperature according to the polynomial fit in Ref. $26 . N_{\text {mol }}$ is the molecular density and $N_{\mathrm{FC}}$ is the density of Franck-Condon atoms. The reduction of the plasma energy due to dissociation, ionization of molecules, and ionization of atoms is described by the pressure sink term,

$$
S^{\mathrm{pe}}=-\phi_{\mathrm{mol}}^{\mathrm{diss}} n N_{\mathrm{mol}}\langle\sigma v\rangle_{\mathrm{mol}}^{\text {diss }}-\phi_{\mathrm{mol}}^{\mathrm{ion}} S_{\mathrm{mol}}^{\mathrm{n}}-\phi_{\mathrm{atom}}^{\text {ion }} S_{\mathrm{FC}}^{\mathrm{n}},
$$

where $\phi$ is the reaction potential. ${ }^{26}$ For hydrogen isotopes, the reaction potential is equal to $4.52 \mathrm{eV}$ for the dissociation of molecules, $18.11 \mathrm{eV}$ for the ionization of molecules, and $13.6 \mathrm{eV}$ for the ionization of atoms.

The neutral source and sink terms are added to the plasma density and electron pressure equations for the coupling of plasma and neutral equations. Since the HESEL is a 2D model with parametrized parallel effects, a correction of the neutral source/sink terms is made, accounting for parallel expansion. This correction is based on the following assumptions. The density source resulting from ionization of neutrals is instantaneously distributed along the flux tube of length $L_{b}=R q$. The size of the neutral beam in the parallel direction is taken as a constant and equal to $w_{\text {beam }}=1 \mathrm{~cm}$. Therefore, the corresponding correction coefficient is introduced, 


$$
K_{\text {toroidal }}=\frac{w_{\text {beam }}}{L_{b}} .
$$

Since the HESEL model imposes periodic boundary conditions in the poloidal direction, the additional correction coefficient is implemented to reproduce only one supersonic molecular beam in the poloidal direction of the tokamak. The corresponding coefficient is written in the following form:

$$
K_{\text {periodic }}=\frac{2 \pi a}{L_{y}},
$$

where $L_{y}$ is the size of the HESEL domain in the poloidal direction. The neutral density source and pressure sink are then multiplied on the normalization coefficient,

$$
K_{\text {norm }}=K_{\text {toroidal }} \cdot K_{\text {periodic }}
$$

while they are added to the HESEL equations.

The resulting system of the plasma and neutrals equations is

$$
\begin{aligned}
& \frac{d n}{d t}+n C(\phi)-C\left(p_{e}\right)=D_{e}(1+\tau) \nabla_{\perp}^{2} n-S_{\|}+K_{\mathrm{norm}} S^{\mathrm{n}} \\
& \nabla\left(\frac{d^{0} \nabla_{\perp} \phi^{*}}{d t}\right)-C\left(p_{e}+p_{i}\right)=\frac{3}{10} D_{i} \nabla_{\perp}^{2} \nabla_{\perp}^{2} \phi^{*} \\
& \quad-\sigma(x) \frac{\nabla_{\perp}^{2} \phi^{*}}{\tau_{n}}+\sigma(x)\left(\mathscr{S}-\alpha\left(\tilde{T}_{e}+\frac{\overline{T_{e}}}{\overline{n_{e}}} \tilde{n}-\tilde{\phi}\right)\right) \\
& \frac{3}{2} \frac{d p_{e}}{d t}+\frac{5}{2} p_{e} C(\phi)-\frac{5}{2} C\left(\frac{p_{e}^{2}}{n}\right) \\
& =D_{e}(1+\tau) \nabla\left(T_{e} \nabla_{\perp} n\right)+\frac{11}{12} D_{e} \nabla\left(n \nabla_{\perp} T_{e}\right) \\
& +D_{e}(1+\tau) \nabla_{\perp} \ln (n) \nabla_{\perp} p_{i}-\frac{3 m_{e}}{m_{i}} \nu_{e i 0}\left(p_{e}-p_{i}\right) \\
& \quad-\sigma(x)\left[\frac{9}{2} \frac{p_{e}}{\tau_{n}}+\frac{T_{e}^{7 / 2}}{\tau_{S H}}\right]-\alpha \overline{T_{e}}\left(\tilde{T}_{e}+\frac{\overline{T_{e}}}{\bar{n}} \tilde{n}-\tilde{\phi}\right)+K_{\mathrm{norm}} S^{p e}
\end{aligned}
$$

$$
\begin{gathered}
\frac{3}{2} \frac{d p_{i}}{d t}+\frac{5}{2} p_{i} C(\phi)-\frac{5}{2} C\left(\frac{p_{i}^{2}}{n}\right)-p_{i} C\left(p_{e}+p_{i}\right) \\
=\frac{5}{2} D_{e}(1+\tau) \nabla\left(T_{i} \nabla_{\perp} n\right)-D_{e}(1+\tau) \nabla\left(n \nabla_{\perp}\right) \ln (n) \nabla_{\perp} p_{i} \\
\left.+2 D_{i} \nabla_{\perp}\left(n \nabla_{\perp}\right) T_{i}\right) \frac{3}{10} D_{i}\left[\left(\partial_{x x}^{2} \phi^{*}-\partial_{y y}^{2} \phi^{*}\right)^{2}+4\left(\partial_{x y}^{2} \phi^{*}\right)^{2}\right] \\
+\frac{3 m_{e}}{m_{i}} \nu_{e i 0}\left(p_{e}-p_{i}\right)+\sigma(x)\left(p_{i} \mathscr{S}-\frac{9}{2} \frac{p_{i}}{\tau_{n}}\right) \\
-p_{i} \alpha\left(\tilde{T}_{e}+\frac{\overline{T_{e}}}{\overline{n_{e}}} \tilde{n}-\tilde{\phi}\right) \\
\frac{\partial N_{\mathrm{mol}}}{\partial t}-D_{\mathrm{mol}} \nabla^{2} N_{\mathrm{mol}}+V \nabla\left(N_{\mathrm{mol}}\right) \\
=-n N_{\mathrm{mol}}\langle\sigma v\rangle_{\mathrm{mol}}^{\mathrm{diss}}-n N_{\mathrm{mol}}\langle\sigma v\rangle_{\mathrm{mol}}^{\text {ion }}, \\
\frac{\partial N_{\mathrm{FC}}}{\partial t}-D_{\mathrm{FC}} \nabla^{2} N_{\mathrm{FC}}+V \nabla\left(N_{\mathrm{FC}}\right) \\
=2 n N_{\mathrm{mol}}\langle\sigma v\rangle_{\mathrm{mol}}^{\mathrm{diss}}-n N_{\mathrm{FC}}\langle\sigma v\rangle_{\text {atom }}^{\text {ion }},
\end{gathered}
$$

where $\phi$ is the plasma potential, $\phi^{*}=\phi+p_{i}$ is the generalized potential, $p_{e}$ and $p_{i}$ are the electron and ion pressure, and $\tau=\frac{T_{i 0}}{T_{e 0}}$ is the ratio of the reference temperatures. The material derivatives are defined as $\frac{d}{d t}=\frac{\partial}{\partial t}+B^{-1}\{\phi, \cdot\}$ and $\frac{d^{0}}{d t}=\frac{\partial}{\partial t}+\{\phi, \cdot\}$ with the anti-symmetric bracket $\{f, g\}=\frac{\partial f}{\partial x} \frac{\partial g}{\partial y}-\frac{\partial f}{\partial y} \frac{\partial g}{\partial x} . \quad C(f)=-\frac{\rho_{s}}{R} \frac{\partial f}{\partial y} \quad$ is the curvature operator.

The fluctuating component of the field $f$ is denoted as $\tilde{f}, \bar{f}$ denotes the poloidal averaged component and $\alpha=\frac{2 T_{e 0}}{\nu_{e i 0} m_{e} L_{b}^{2} \Omega_{i 0}}$ is the drift-wave coefficient presenting only in the edge region, where $m_{e}$ is the electron mass.

$\mathscr{S}=\frac{e n_{0}\left\langle c_{s}\right\rangle}{L_{c}}\left[1-\exp \left(\Lambda-\frac{e\langle\phi\rangle}{\left\langle T_{e}\right\rangle}\right)\right]$ is the sheath damping term, where $n_{0}$ is the characteristic electron density and $\Lambda=\log \left(\sqrt{\frac{m_{i}}{2 \pi m_{e}}}\right)$ is the Bohm potential.

The diffusion coefficient for particular neutral species is proportional to the thermal energy,

$$
D_{n}=\frac{1}{2} \frac{T_{n}}{m_{n} N_{n}\langle\sigma v\rangle} .
$$

The symbol $n$ denotes the molecules (mol) or atoms (FC), respectively.

The injection neutral source is set by the Dirichlet boundary conditions at the outer radial boundary and zero gradient Neumann boundary conditions are applied at the inner radii boundary. The initial profile of the injected beam is approximated by a Gaussian function. Owing to the deeper penetration of SMBI compared to $\mathrm{GP},{ }^{32}$ the recycling effect is smaller and not included in the model.

The model is implemented in the BOUT ++ framework, ${ }^{33}$ using the implicit time solver and the fourth order differential scheme.

\section{SIMULATION SETUP}

Deuterium plasmas and deuterium molecular beam injections are considered in simulations. The typical initial parameters for the HESEL simulations are taken for a medium-sized tokamak. The reference parameters in this work are: major radius $R=1.8 \mathrm{~m}$, minor radius $a=0.5 \mathrm{~m}$, parallel connection length to the divertor targets $L_{c}=15.0 \mathrm{~m}$, and magnetic field strength $B_{0}=2 \mathrm{~T}$. To investigate SMBI in different plasma conditions, the safety factor varies in the range $q=3-7.5$ which reflects in the low plasma density and temperature for the low safety factor and high plasma parameters for the high safety factor. Gyro-Bohm normalization parameters are used in the model, namely the characteristic electron density $n_{0}=1.5 \times 10^{19} \mathrm{~m}^{-3}$ and the characteristic electron and ion temperatures $T_{e 0}=T_{i 0}=20 \mathrm{eV}$ are taken for this simulations. The plasma parameters are the following: the characteristic ion gyro-frequency $\Omega_{i 0}=7.48 \times 10^{7} \mathrm{~s}^{-1}$ and the cold-ion hybrid thermal gyro-radius $\rho_{s}=4.13 \times 10^{-4} \mathrm{~m}$. The domain is $11 \times 11 \mathrm{~cm}$. The width of the edge region (excluding the forcing region with prescribed profiles) is $4 \mathrm{~cm}$, the width of the SOL region is $5 \mathrm{~cm}$, and the width of the wall region, which acts as a damping layer, is $1 \mathrm{~cm}$. The spatial resolution is $\mathrm{dx}=\mathrm{dz}=5.2 \times 10^{-3} \mathrm{~m}$.

A supersonic molecular beam is injected into a statistically steady-state turbulent plasma and an injection lasts until the end of simulations. Data for the analysis are taken when the plasma-neutral interactions reach conditions close to the steady-state, corresponding to $0.8 \mathrm{~ms}$ after the start of SMBI.

The diffusion coefficients are $D_{\mathrm{mol}}=0.03 \mathrm{~m}^{2} \mathrm{~s}^{-1}$ and $D_{\mathrm{FC}}$ $=33.3 \mathrm{~m}^{2} \mathrm{~s}^{-1}$ for molecules and atoms, respectively. 
TABLE I. Range of scanning simulation parameters

\begin{tabular}{lcc}
\hline \hline Parameter & & Range \\
\hline $\mathrm{q}$ & Safety factor & $3-7.5$ \\
$\left\langle N_{\mathrm{mol}}\right\rangle\left(\mathrm{m}^{-3}\right)$ & Density of the beam & $0-8 \times 10^{22}$ \\
$V\left(\mathrm{~m} \mathrm{~s}^{-1}\right)$ & Velocity of the beam & $10-10000$ \\
$\mathrm{w}(\mathrm{cm})$ & Width of the beam & $0.1-11$ \\
\hline \hline
\end{tabular}

The initial parameters of the molecular beam, namely, beam density, velocity, and width are varied in order to estimate how the penetration of neutrals depends on the injection parameters. The parameter ranges are presented in Table I. These parameters are compatible with experimental values.

\section{RESULTS}

The results represent the output of self-consistent simulations of turbulent fusion plasma and the neutral beam injected into the plasma.
As seen from Eqs. (10)-(15), plasmas and neutral fields are related to the corresponding source terms which are functions of neutral densities (molecules and atoms), plasma density, and electron temperature. Figure 2 shows how the molecular beam density and density source correlate with the plasma density and temperature fluctuations. As seen from the figure, the shape of the molecular beam is affected by the spatial structures of the plasma density. The locations of the density source correlate with spots of high plasma temperature.

\section{A. Scan of beam density}

For the beam density scan, the velocity of the beam is set to $V=$ $1000 \mathrm{~m} / \mathrm{s}$ and the width of the beam is $w=2 \mathrm{~cm}$. The influence of SMBI on plasma results in a higher averaged plasma density and lower averaged plasma temperature. Since the amplitude of the density source grows with the increase in the amount of injected particles, the influence of neutral injection on plasma becomes more pronounced with the increase in the neutral beam intensity. Temporally averaged (over around 27 autocorrelation times) plasma densities and
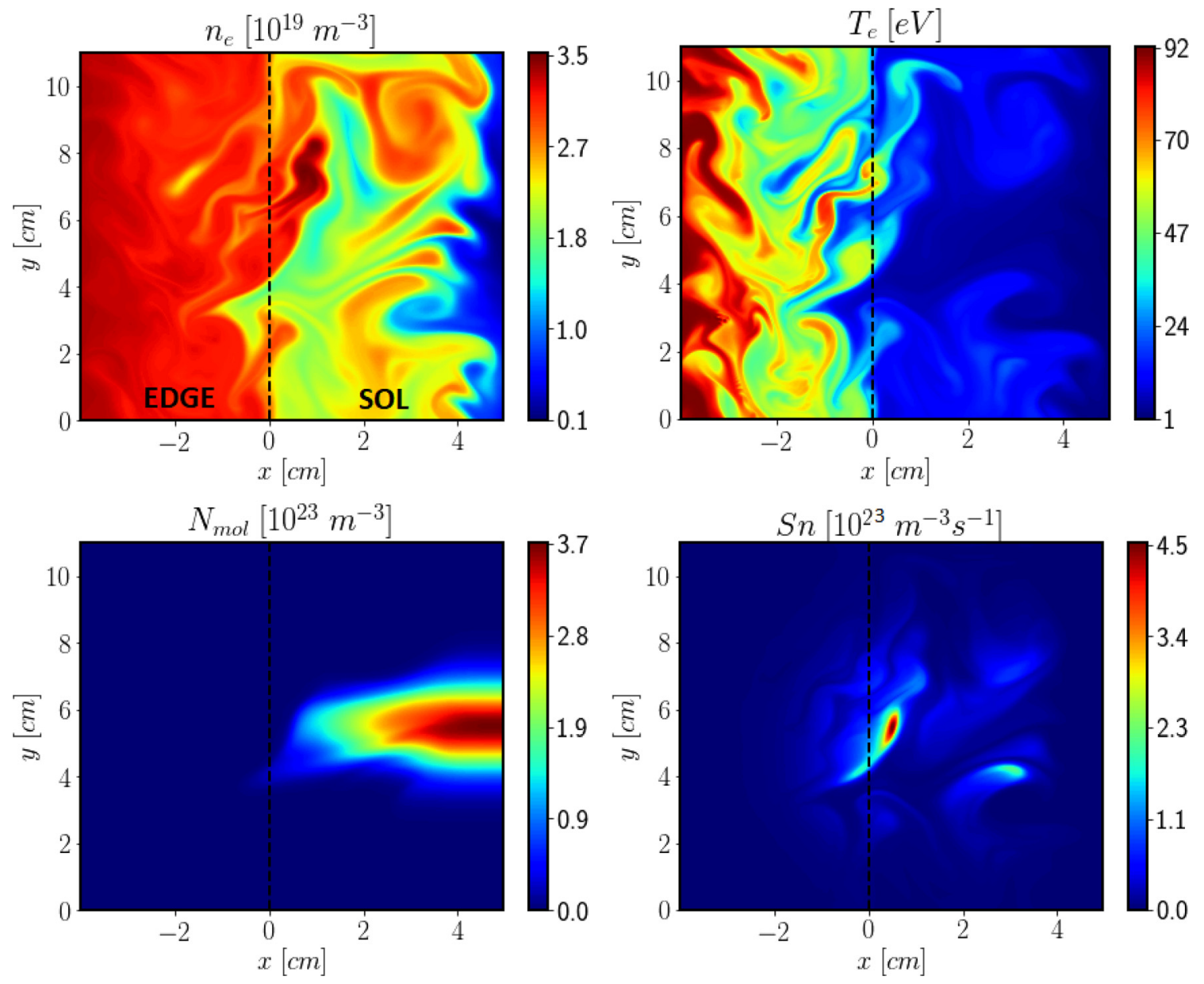

FIG. 2. On the top: plasma density (left) and electron plasma temperature (right). On the bottom: Molecular beam density (left) and density source produced by ionization of neutrals (right). All plots correspond to $t=1.068 \mathrm{~ms}$ and the initial parameters $\mathrm{q}=5.5, \mathrm{~N}_{\text {mol }}=7.4 \times 10^{22} \mathrm{~m}^{-3}, \mathrm{~V}=1000 \mathrm{~m} / \mathrm{s}, \mathrm{w}=2 \mathrm{~cm}$. The position of LCFS corresponds to $x=0$. 
temperatures for the two injection rates are compared with the simulations without SMBI in Fig. 3. As can be seen, the influence of the molecular beam on the plasma dynamics is different for low $\left(\left\langle N_{\text {mol }}\right\rangle_{y}=1.3 \times 10^{22} \mathrm{~m}^{-3}\right)$ and high $\left(\left\langle N_{\mathrm{mol}}\right\rangle_{y}=8.7 \times 10^{22} \mathrm{~m}^{-3}\right)$ density injections. The high neutral injection forms the peak of plasma density in the poloidal center of the domain in few centimeters inside the separatrix, which indicates the penetration of neutrals inside the edge region and ionization there. In the SOL region, the plasma density increases not just on the beam penetration path but over the entire poloidal direction in both cases of neutral injection. It should be noted that the higher plasma density in the upper part of the domain in the SOL region is related to blobs dynamic. It can be seen on the plot demonstrating the plasma density without SMBI that the density in the right upper corner of the SOL region is slightly higher. In the case of the high neutral injection, the bump of the plasma density at the middle of the SOL region appears due to strong concentration of neutrals in this area. The electron temperature strongly decreases on the beam penetration path in the case of high density neutral beam injection. To see this effect clearer, the average temperature is shown only in the SOL region in Fig. 3. This effect is not observed for beams with intensity smaller than $\left\langle N_{\text {mol }}\right\rangle_{y}=6 \times 10^{22} \mathrm{~m}^{-3}$ and this indicates that the energy required for the ionization and dissociation of such beams does not exceed the turbulence plasma energy flux from the edge region. The "hole" in the plasma temperature field is observed only in the SOL region; however, the averaged decrease in the plasma temperature in the edge region takes place with neutral beam injection higher than $\left\langle N_{\mathrm{mol}}\right\rangle_{y}=6 \times 10^{22} \mathrm{~m}^{-3}$.

An analysis of the density source [Eq. (5)] produced by ionization of neutrals allows for estimating the neutral penetration depth and the fueling efficiency of SMBI. The amplitude of the density source and the ionization frequency in the edge region grows with the beam intensity. Figure 4 demonstrates that the temporally and poloidally averaged density source profile shifts toward the LCFS with the increase in the amount of injected particles. This can be explained by the drop of plasma temperature and consequently decrease in ionization rates in the SOL. The density source in the edge region is produced mostly by ionization of atoms rather than molecules. However, the ratio of molecules to atoms in the edge region is not constant and grows with intensity of the beam. For the low density neutral injection, the percentage of molecules ionized in the edge compared to the total ionization in the edge is less than $1 \%$, while for the high density injection, this value increases up to $6 \%$.

We compared the density source with the particle sink in the SOL region described by Eq. (4). The particle sink in the HESEL model
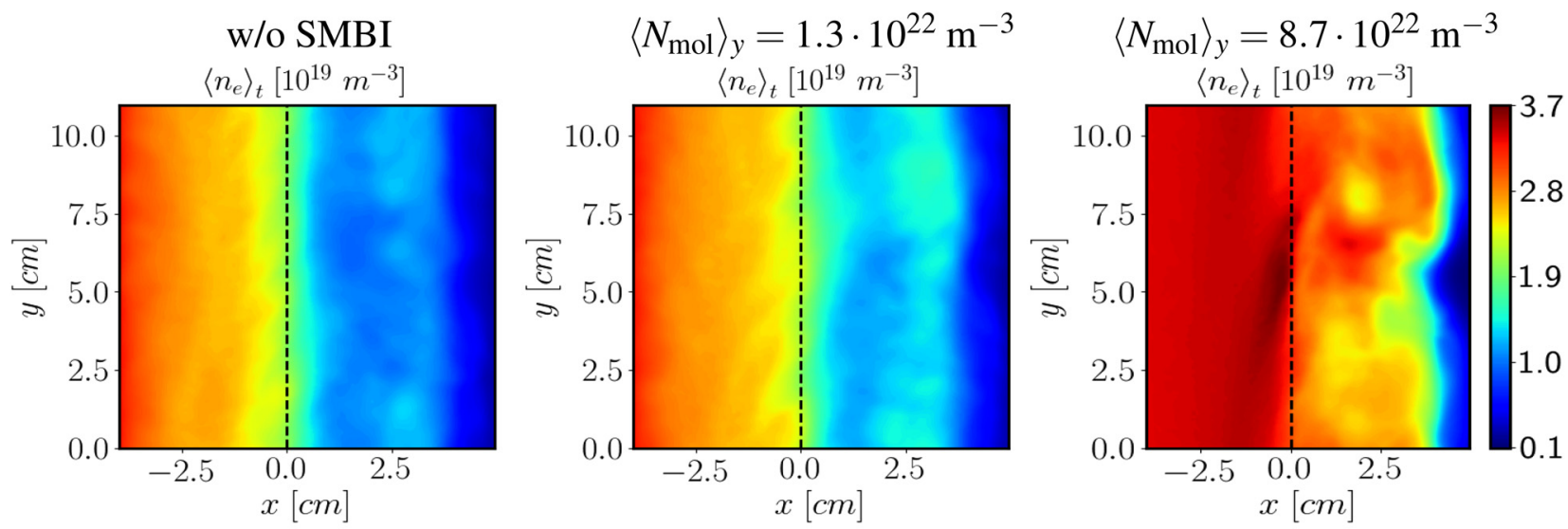

Temperature is shown only in the SOL region
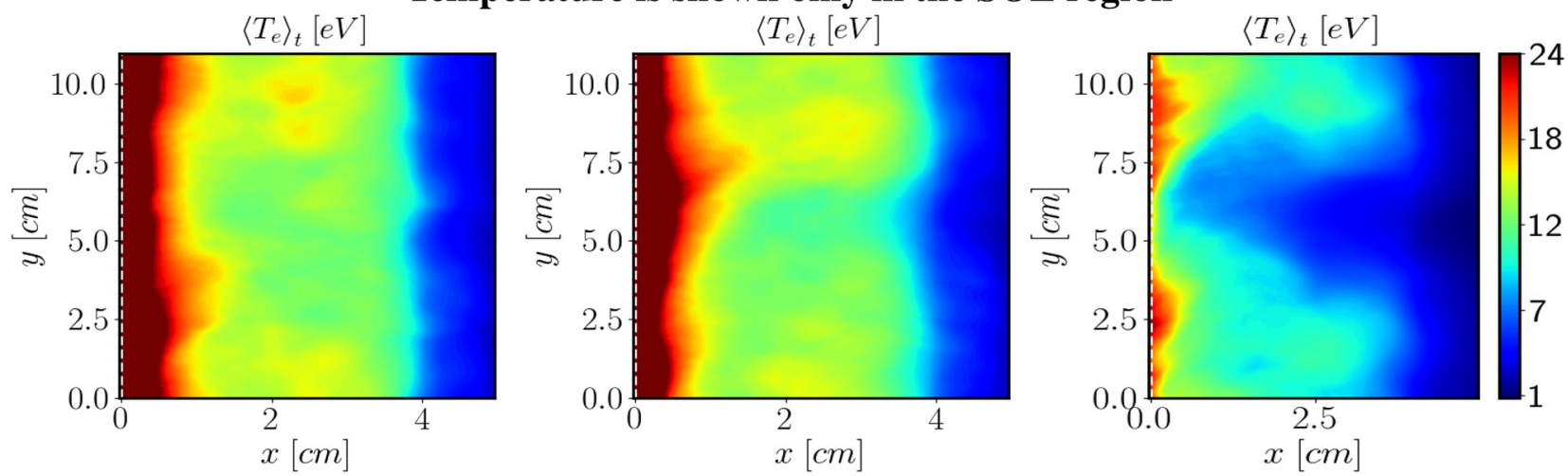

FIG. 3. Temporally averaged plasma density and temperature in simulations with different densities of the supersonic molecular beam. Note that the temperature is shown only in the SOL that is done for adjusting a convenient color-bar scale. Simulations are performed with $q=5.5$. $Y$ is equivalent to the poloidal direction, $\mathrm{x}$ is a radial direction. Time averaging is performed over $0.27 \mathrm{~ms}$ when plasma and molecular beam reach statistically quasi-steady state conditions. 


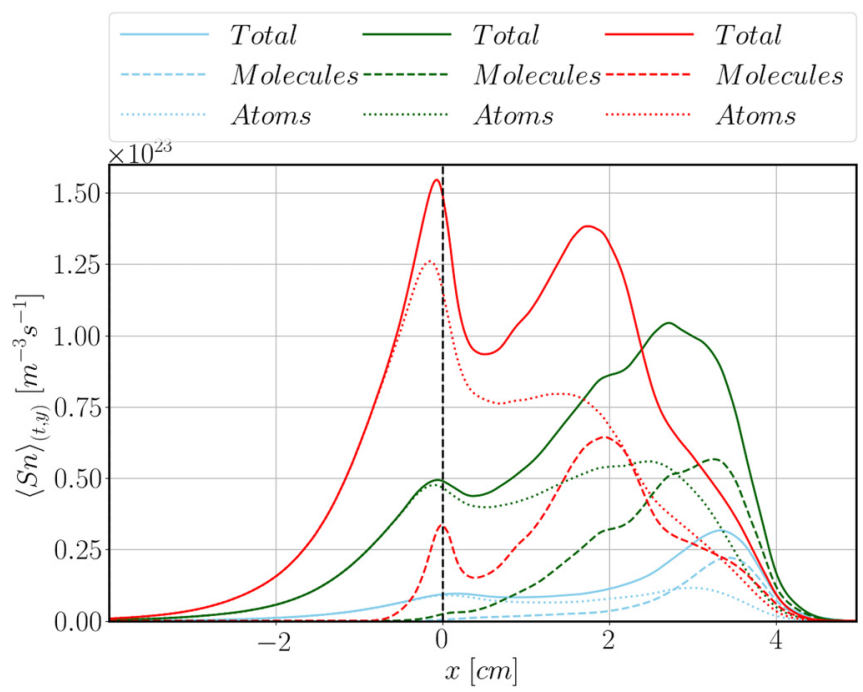

FIG. 4. Temporally and poloidally averaged density source profiles resulting from ionization of neutrals are shown for different densities of injected beams. Contributions of the molecules and atoms to the total density source are shown by the dashed and dotted lines, respectively. Simulations are performed with $q=5.5$.

is defined through the damping term, which depends on the plasma temperature, parallel Mach number, safety factor, and major radius of a tokamak [see Eq. (2)]. This comparison demonstrates that the source produced by injection of the beam with density $\left\langle N_{\text {mol }}\right\rangle_{y}=$ $6 \times 10^{22} \mathrm{~m}^{-3}$ is of the same order of magnitude as the particle sink. The lower density beam injection produces a source smaller than the plasma sink, while in the case of higher density injection, the source exceeds the particle losses. The decrease of the plasma temperature due to ionization of neutrals results in a longer particle lifetime in the SOL region and enhanced probability of ions to diffuse inside the confined region.

In the edge region, the integrated density source is compared with the plasma source prescribed in the HESEL forcing region described in Sec. II. The compared quantities are the sources defined by Eqs. (1) and (5) integrated over the edge region,

$$
\begin{gathered}
\text { Forcing }=\int_{\text {edge }} S_{\text {force }} d x d y \\
\text { Fuelling }=\int_{\text {edge }} S^{\mathrm{n}} d x d y
\end{gathered}
$$

and shown in Fig. 5. As can be seen, the fueling by the neutral beam with density $\left\langle N_{\text {mol }}\right\rangle_{y}=6 \times 10^{22} \mathrm{~m}^{-3}$ is comparable with the forcing. Summarizing all observations, the beam density $\left\langle N_{\text {mol }}\right\rangle_{y}$ $=6 \times 10^{22} \mathrm{~m}^{-3}$ can be defined as the "threshold density." To define the threshold molecular beam density, we compared the density sources produced by ionization of neutrals with the plasma density sources at the core, i.e., fueling, and particle losses in the SOL for all considered molecular beam densities listed in Table I. The neutral injection below the threshold density forms the density source much smaller compared to the plasma source at the core and the particle sink in the SOL region. The decrease in the plasma temperature caused by such injection is negligible. In tokamak experiments, the threshold neutral density should be compared with the plasma density source resulting from PI or NBI, which provide the core fueling.

In Ref. 23, the possibility of replacing the force profile with the density source was demonstrated. Such simulations are beneficial in terms of the analogy with the real experiments where the plasma density source is due to the ionization of neutrals. However, removing the forcing is not trivial for SMBI simulations, as the density source does not evenly distribute across the plasma surface.

It is difficult to make a prediction of the density source in the edge region for the particular beam density as the neutral penetration depends on the plasma dynamics. The scan of the safety factor in HESEL simulations allows for obtaining different plasma conditions. The plasma diffusion coefficients, damping terms, and the drift-wave coefficient are dependent on the safety factor. The resulting plasma profiles are defined by non-linear interactions of these terms in the HESEL model. The plasma profiles before the neutral injection corresponding to simulations with different safety factors are shown in Fig. 6. The values of the plasma density and plasma temperature at the separatrix are in the range of $n^{\mathrm{LCFS}}=1.7-2.2 \times 10^{19} \mathrm{~m}^{-3}$ and $T_{e}^{\mathrm{LCFS}}$ $=36-46 \mathrm{eV}$, respectively. The beam density scan in various plasma
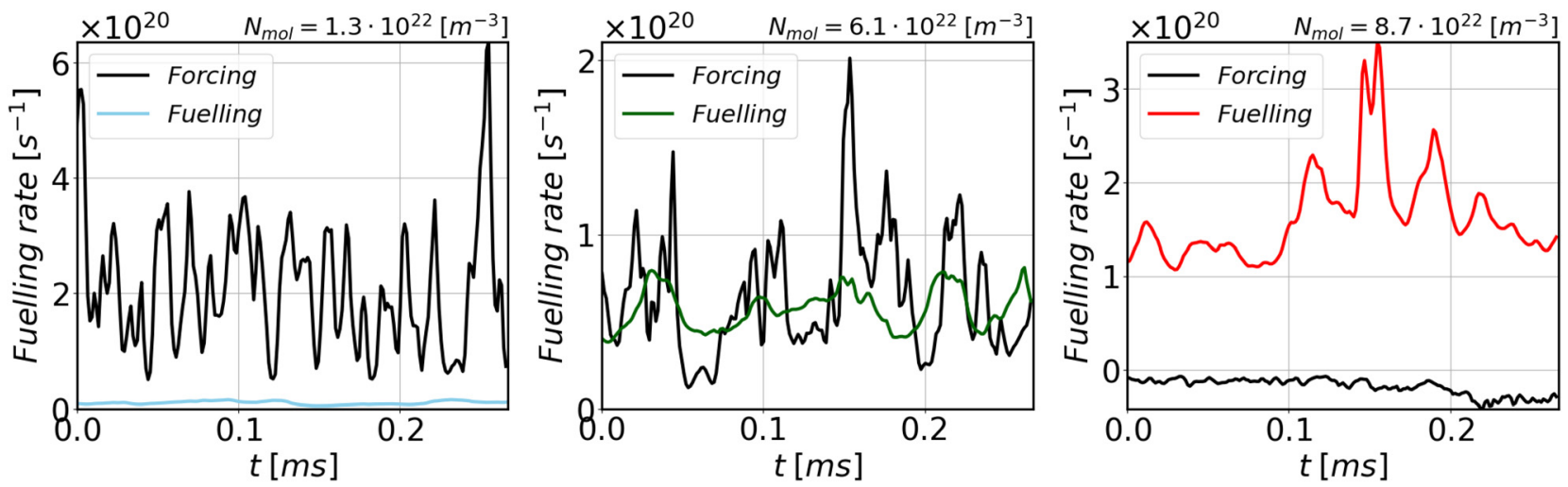

FIG. 5. Comparison of the prescribed forced density source (forcing), which represents a source of particles from the core region, with the density source resulting from the ionization of neutrals (fueling). Simulations are performed with $q=5.5$. 

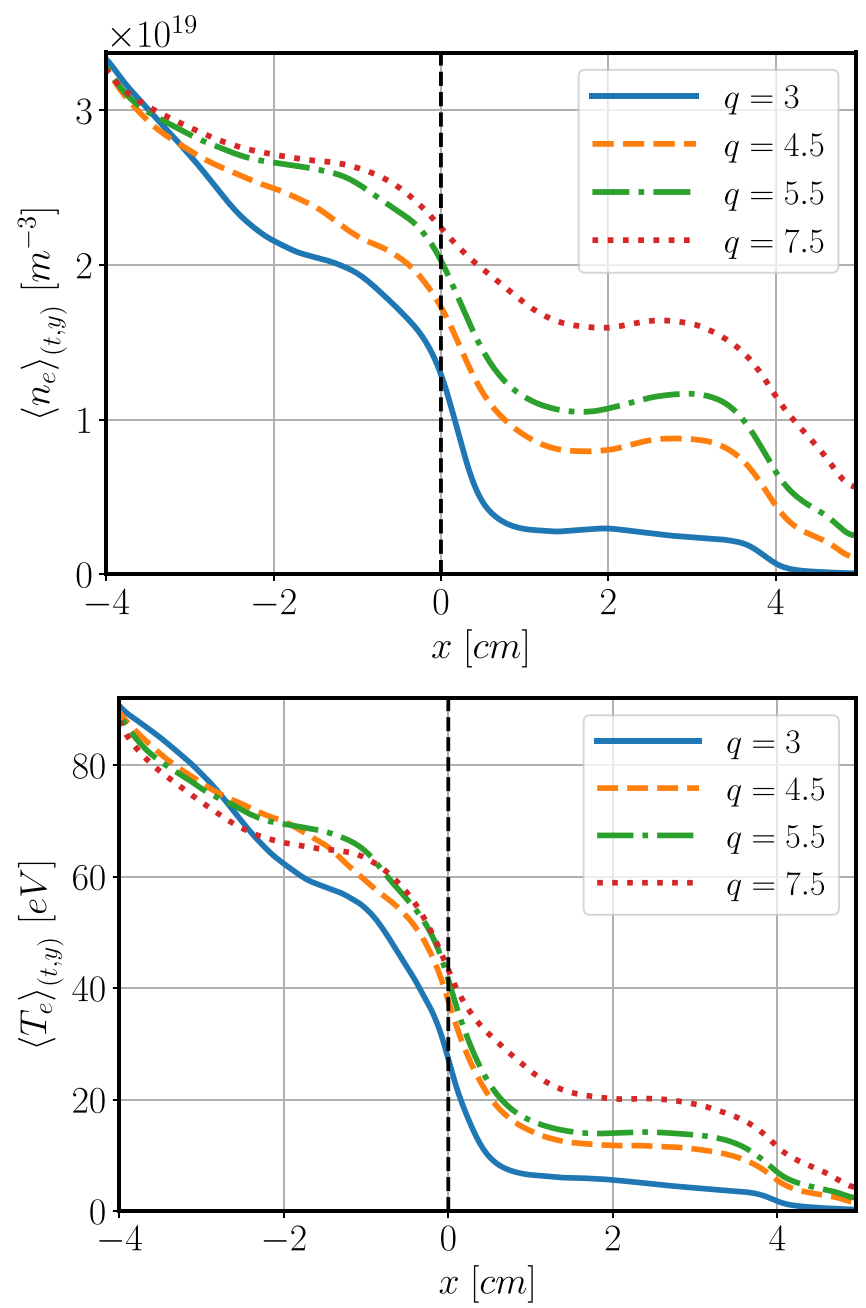

FIG. 6. Plasma profiles before the neutral injection corresponding to simulations with different safety factors.

conditions is performed with focusing on how the fueling efficiency depends on the beam parameters in different plasma conditions.

However, it is not straightforward to correctly estimate the fueling efficiency, i.e., the ratio of the density increment to the number of injected molecules, in terms of the HESEL model. The inward particle transport, which was observed in experiments with $\mathrm{SMBI},{ }^{2}$ is reproduced in the HESEL model in agreement with turbulent equipartition models; ${ }^{34}$ however, the plasma density level cannot be dynamically adjusted. Thus, the density increase after SMBI cannot be modeled in total, only the alteration of the plasma properties in the SOL and a few centimeters inside the separatrix. Therefore, the ionization efficiency $(\eta)$ is defined as the ratio of the density source in the edge region to the total density source over the whole domain,

$$
\eta=\frac{\int_{\text {edge }} S^{\mathrm{n}} d x d y d t}{\int_{\text {total }} S^{\mathrm{n}} d x d y d t} .
$$

Note that while the fueling efficiency includes induced changes to confinement under fueling, the ionization efficiency gives the plasma production efficiency. The ionization efficiency allows an estimation of the feasibility of SMBI to effectively fuel the plasma in the assumption that only the ionization in the edge region can effectively increase the plasma density, while the ionization in the SOL is ineffective due to parallel losses along the open magnetic field lines.

Scaling of the ionization efficiency as a function of beam intensity is shown in Fig. 7. There is no universal dependency and the fueling efficiency varies depending on the plasma conditions. The simulations with $q=3$ are characterized by low initial plasma density and temperature (Fig. 6). The injected neutrals penetrate through the SOL region and ionize mostly in the edge even in the case of the low density injection. The increase in the molecular injection density leads to the increase in the averaged plasma density and enhanced plasma-neutral interaction in the SOL region. Thereby the decrease in the ionization efficiency with the increase in the molecular beam intensity in simulations with $q=3$ is understandable. The maximum intensity of the molecular beam is restricted by the HESEL boundary conditions and the prescribed profile of the plasma density in the inner edge (forcing) region. In order to keep the working parameters of the HESEL model, which are required for the correct description of the plasma dynamics, the density source produced by the ionization of injected neutrals should not lead to an excess of the prescribed density level in the inner edge region. In the simulations with $q=4.5$, the plasma dynamics in the middle of the SOL region plays the essential role in the radial distribution of the density source as the main fraction of the neutrals is ionized there. The efficiency is almost independent of the beam density in the range of the considered parameters for the scan with $q=4.5$. The threshold beam density for this plasma condition is $\left\langle N_{\text {mol }}\right\rangle_{y}=4 \times 10^{22} \mathrm{~m}^{-3}$ and the increase in the efficiency with the beam density above this value is expected due to strong cooling of the plasma in the SOL region. In simulations with $q=5.5$, the efficiency exponentially increases with the molecular density. This behavior is likely caused by the strong decrease in the plasma temperature in the SOL region which is observed only if the density of injection beam is

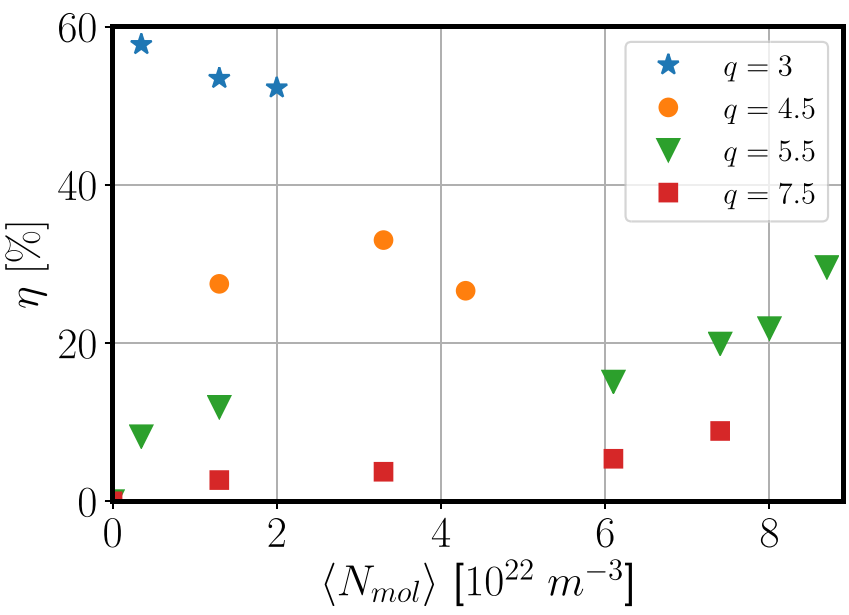

FIG. 7. Ionization efficiency $(\boldsymbol{\eta})$ as a function of the averaged molecular beam density for simulations with different safety factors. 
high enough. In simulations with $q=7.5$, the efficiency only slightly increases over the full range of considered parameters.

\section{B. Scan of beam velocity}

A scan of the molecular beam velocity is carried out to s tudy how the increase in the directed beam velocity affects the neutral penetration depth. A scan of the beam velocity is performed for small flux (smaller than the threshold density) $\left\langle\Gamma_{\text {inj }}\right\rangle_{q 5.5}^{\text {small }}=1.3 \times 10^{25} \mathrm{~m}^{-2} \mathrm{~s}^{-1}$ and for large flux (larger than the threshold density) $\left\langle\Gamma_{\text {inj }}\right\rangle_{q 5.5}^{\text {large }}=7.4$ $\times 10^{25} \mathrm{~m}^{-2} \mathrm{~s}^{-1}$ with the safety factor $q=5.5$. In addition, a scan for the simulations with low and high safety factor is performed with the fluxes $\left\langle\Gamma_{\text {inj }}\right\rangle_{q 3}^{\text {large }}=2 \times 10^{25} \mathrm{~m}^{-2} \mathrm{~s}^{-1}$ and $\left\langle\Gamma_{\text {inj }}\right\rangle_{q 7.5}^{\text {small }}=7.4$ $\times 10^{25} \mathrm{~m}^{-2} \mathrm{~s}^{-1}$, respectively. The width of the molecular beam is $w=2 \mathrm{~cm}$. We vary the beam density to keep the injected flux constant.

The decrease in the averaged electron temperature of the beam propagation front in the SOL region is observed in simulations with $\left\langle\Gamma_{\text {inj }}\right\rangle_{q 5.5}^{\text {large }}$. The increase in the beam velocity leads to the propagation of the cold front deeper into the plasma. Unlike in the beam intensity scan, the amplitude of the density source in the SOL region does not rise with increasing beam velocity and the absolute decrease in the temperature becomes smaller with increasing injection velocity. Regarding the plasma density, the injection of the beam with higher velocity leads to increasing plasma density and formation of a poloidally localized density source inside the edge region. For small injection flux at the same plasma conditions, no visible changes in the averaged plasma fields are observed.

$2 \mathrm{D}$ plots of the neutral fields and the density source in simulations with $\left\langle\Gamma_{\text {inj }}\right\rangle_{q 5.5}^{\text {large }}$ are shown in Fig. 8. Each plot shows the time averaged field normalized to its maximum. The molecules penetrate deeper inside the plasma with the increase in the direct velocity as the convection determines their transport. For the atoms, convection and diffusion are comparable as they appear from the Péclet number, which is around 290 for molecules and 0.3 for atoms. The thermal energy of $2 \mathrm{eV}$ corresponds to a thermal velocity approximately 14000 $\mathrm{m} / \mathrm{s}$. The radial extent of the atomic density distribution demonstrates the influence of the injection velocity on the penetration of atoms even with the amplitude of the direct velocity lower than the thermal velocity. The density source shifts deeper inside the plasma with increase in the injection velocity. The radially narrow localization of the density source produced by the beam with the low injection velocity is understandable as the plasma density and temperature are low in the far SOL region. Therefore, the ionization frequency is relatively small and the density source is created owing to the high molecular injection density. With the increase in the injection velocity, neutrals penetrate deeper inside the plasma where plasma fluctuations enhance the ionization reaction rate and the broad distribution of the density source is seen on the time averaged plot. The reduction of the density source in the SOL region compared to the edge region is observed with further increase in the beam velocity. This is likely related to lower plasma density and temperature in the SOL region compared to the edge.

The tendency of the deeper neutral penetration with increase in the beam velocity is observed for both small and large injection fluxes in the same plasma conditions as shown in Fig. 9. Since the low injection flux $\left\langle\Gamma_{\text {inj }}\right\rangle_{q 5.5}^{\text {small }}$ does not induce a noticeable decrease in plasma temperature in the SOL region, the increase in the neutral penetration depth with increase in the beam velocity is governed by the convection transport. In the case of the large injection flux both effects convection and reduction of plasma temperature lead to enhanced penetration of the neutrals into the plasma.

Regarding comparison of fueling and forcing, for the same injection flux the molecular beam with low injection velocity cannot provide the density source at the edge region comparable to the plasma forcing as can be seen in Fig. 10.

The ionization efficiency as a function of the molecular beam velocity is shown in Fig. 11. In the case of simulations with $q=3$, the temperature and density are low and the difference between density source profiles is negligible for the velocities of 400 and $1000 \mathrm{~m} / \mathrm{s}$. Unlike in the beam intensity scan, the dependency of the ionization efficiency on the molecular beam velocity can be described by the same simple functional dependency $\eta=a \cdot V^{b}$ for all considered plasma conditions and injection rates. However, with the increase of the plasma density and temperature or decrease in the injection flux, the efficiency grows slowly with the increase in the beam velocity. The approximation coefficients are summarized in Table II.

\section{Scan of beam width}

A scan of the beam width is performed for the small $\left\langle\Gamma_{\text {inj }}\right\rangle_{q 5.5}^{\text {small }}$ $=1.3 \times 10^{25} \mathrm{~m}^{-2} \mathrm{~s}^{-1}$ and large $\left\langle\Gamma_{\text {inj }}\right\rangle_{q 5.5}^{\text {large }}=7.4 \times 10^{25} \mathrm{~m}^{-2} \mathrm{~s}^{-1}$ fluxes and with low $\left\langle\Gamma_{\text {inj }}\right\rangle_{q 3}^{\text {large }}=2.0 \times 10^{25} \mathrm{~m}^{-2} \mathrm{~s}^{-1}$ and high $\left\langle\Gamma_{\text {inj }}\right\rangle_{q 7.5}^{\text {small }}=7.4 \times 10^{25} \mathrm{~m}^{-2} \mathrm{~s}^{-1}$ safety factors. The total injection density and injection velocity of $V=1000 \mathrm{~m} / \mathrm{s}$ are kept constant. The beam width of $11 \mathrm{~cm}$ represents a uniform density injection level over the domain and can be considered as a homogeneous beam with infinite width.

The results indicate that the beam density rather than the width determines the beam penetration and fueling efficiency. As for the velocity scan there are no differences in the observed plasma profiles in the scan with the small injection flux $\left\langle\Gamma_{\text {inj }}\right\rangle_{q 5.5}^{\text {small }}$. The difference in plasma profiles with the $\left\langle\Gamma_{\text {inj }}\right\rangle_{q 5.5}^{\text {large }}$ is moderate; however, the deeper penetration and stronger influence of the narrow beam $(w=0.1 \mathrm{~cm})$ in the edge region are observed. The poloidally localized density source appears inside the edge region only in the case of the narrow beam injection. In the SOL region, plasma profiles are similar and independent of the beam width. However, the hole in plasma temperature, created by the narrow beam in the SOL region, is visible and is likely the reason for its deeper penetration.

Comparison of the fueling from the density forcing with the neutral source resulting from ionization of neutrals shows that the increase in the beam width decreases the ratio of the neutral density source to the plasma source in the edge region.

The ionization efficiency as a function of the beam width is shown in Fig. 12 and the approximation coefficients are summarized in Table III. As can be seen, the efficiency is almost independent of the beam width in the low density and low temperature plasma conditions or with small neutral injection flux. The efficiency decreases with the beam width in the case of large injection flux or injection into high pressure plasma. 

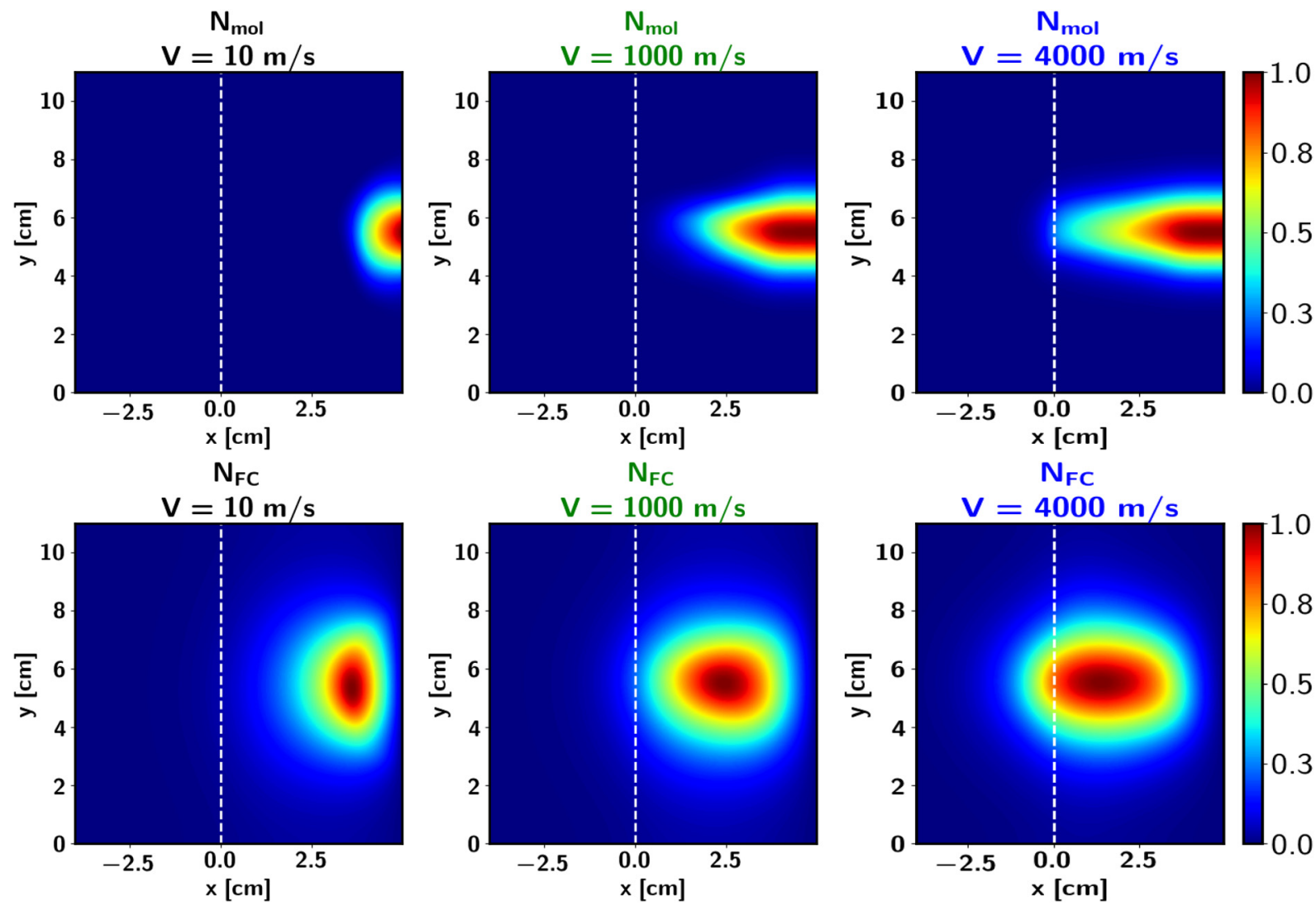

Sn
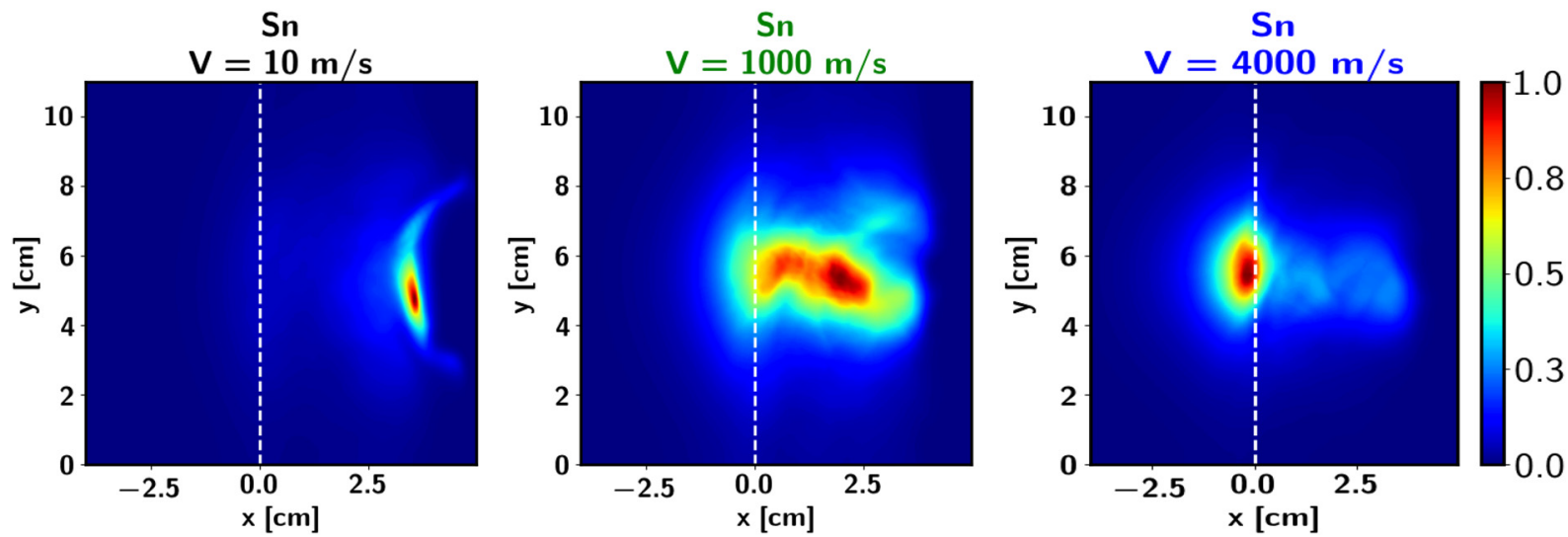

FIG. 8. Temporally averaged density of injected molecules $\left(N_{m o l}\right)$, density of Franck-Condon atoms $\left(N_{F C}\right)$ and density source $(S n)$. For convenience each field is normalized on its maximum. Simulations are performed with $\left\langle\Gamma_{\text {ini }}\right\rangle_{a 55}^{\text {arge }}=7.4 \times 10^{25} \mathrm{~m}^{-2} \mathrm{~s}^{-1}$ and injection velocity of molecules $V=10 \mathrm{~m} / \mathrm{s}$ (first column), $V=1000 \mathrm{~m} / \mathrm{s}$ (second col$u m n$ ), and $V=4000 \mathrm{~m} / \mathrm{s}$ (third column).

\section{Influence of turbulence on the SMBI fueling and influence of SMBI on plasma dynamic}

In order to study the influence of plasma fluctuations on the penetration of the supersonic molecular beam, we compared the simulations performed with the full dynamic model described above (denote this case as "dynamic") with the simulations using the mean-field approximation (denote this case as "steady"), where dynamic plasma profiles are replaced by time averaged fields. The time averaged density source profiles are shown in Fig. 13 and correspond to the case with the narrow beam $w=0.1 \mathrm{~cm}$ with the density $\left\langle N_{\text {mol }}\right\rangle_{y}=7.4$ $\times 10^{22} \mathrm{~m}^{-3}$ and velocity $V=1000 \mathrm{~m} / \mathrm{s}$ injected into the plasma with $q=5.5$. As seen from the figure, the discrepancy between the profiles is not pronounced and the mean (averaged in time and space) values of the density source differ by $1.8 \%$ for these two models. We 

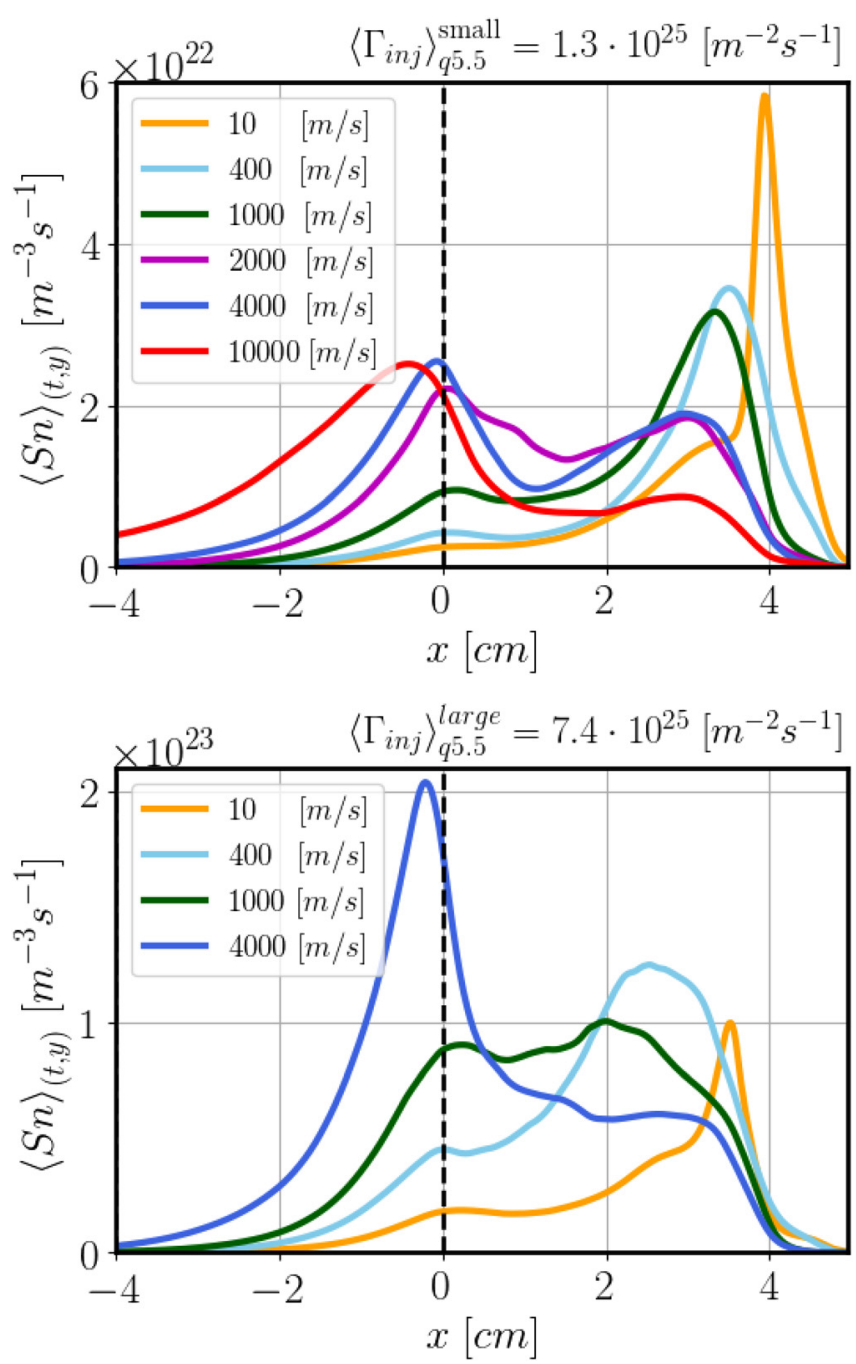

FIG. 9. The averaged density source profiles for simulations with different injection velocities. performed a similar analysis for the other considered cases of neutral injection and the deviation of the mean values obtained in the steady simulations from the dynamic case does not exceed 3\%. Such a slight effect of turbulence on the SMBI fueling can be explained by the high direct velocity of molecules leading to a mean free path much longer compared to the turbulence correlation length.

The fluctuation level for the plasma density fluctuations is defined by the equation,

$$
\zeta_{n} e=\frac{\sqrt{\left\langle\left(n_{e}-\left\langle n_{e}\right\rangle_{(t, y)}\right)^{2}\right\rangle_{(t, y)}}}{\left\langle n_{e}\right\rangle_{(t, y)}}
$$

and shown in Fig. 14 for different plasma conditions. We can see that the variation of $\mathrm{q}$ affects the plasma density fluctuation level; however, it is not straightforward to predict how exactly, since the HESEL is a nonlinear model and the different parameters, namely, diffusion coefficients, sheath damping term, and drift-wave coefficient have a dependence on the safety factor. However, the common features for all cases, that the fluctuation level for the plasma density fluctuations in the edge is around $20 \%$ and increases in the SOL. We have observed that SMBI causes modification of plasma dynamic. As seen in Fig. 15 the increase in the molecular density leads to decreasing of the fluctuation level. It should be noted that the molecular density should be higher than the threshold value to significantly affect the turbulence. As demonstrated in Fig. 15, the injection of the wide neutral beam decreases the fluctuation level stronger compared to the same density narrow beam and no effect of the beam velocity on the fluctuation level is observed. We want to emphasize that the poloidal averaging over the domain is applied in calculations of the fluctuation level. It can explain that despite the deeper penetration and stronger influence on plasma profiles the reduction of the plasma fluctuation level is less for the narrower beam than for the wide beam. The suppression of turbulence is caused by the decrease in plasma pressure gradients. Corresponding plots and additional investigation of the influence of SMBI on plasma turbulence can be found in Ref. 35 .

\section{DISCUSSION}

We want to discuss the obtained results in comparison with findings from previous numerical models applied for investigations of
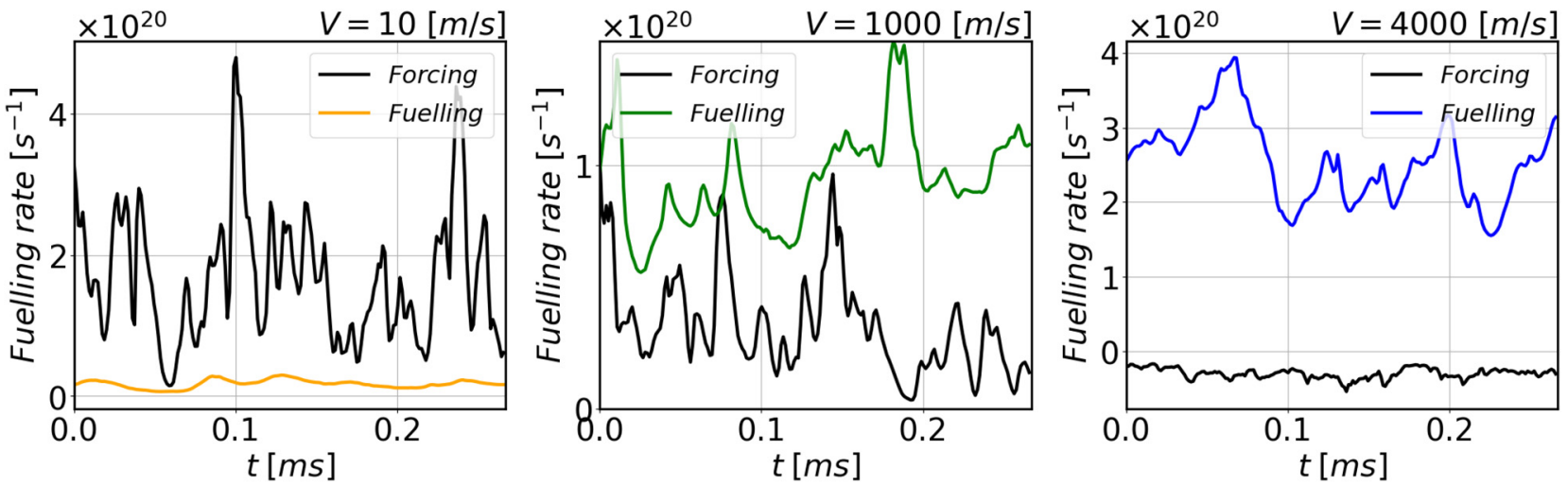

FIG. 10. Comparison of the prescribed forced density source (forcing) which represents a source of particles from the core region with the density source resulting from the ionization of neutrals (fueling). Simulations are performed with $\left\langle\Gamma_{\mathrm{inj}}\right\rangle_{q 5.5}^{\text {large }}=7.4 \times 10^{25} \mathrm{~m}^{-2} \mathrm{~s}^{-1}$. 


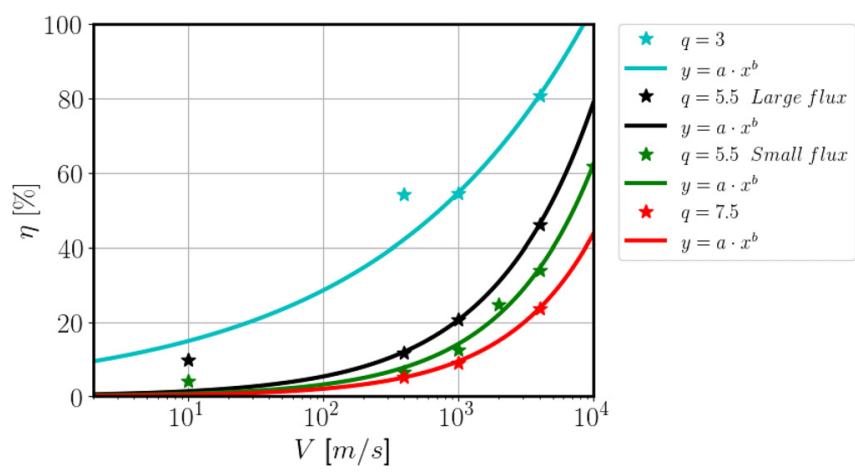

FIG. 11. Ionization efficiency as a function of molecular beam velocity.

SMBI. A wide range of questions regarding SMBI has been investigated with the trans-neut module of the BOUT ++ code. $^{13-15,36-38}$ This model couples plasma density, heat, and momentum transport equations together with neutral density and momentum transport equations for both molecules and atoms. Plasma neutral interactions include dissociation, ionization, recombination and charge-exchange reactions. Plasma dynamics is not included in this model.

Similar to our results, the trans-neut module has demonstrated the increase in the SMBI fueling efficiency with the increase in the molecular beam velocity; however, the penetration depth does not vary much once the SMBI injection density is larger than the critical value $N_{\text {mol }}=0.5 \times 10^{19} \mathrm{~m}^{-3}$. In the case of injection density below the critical value, the molecular injection rate and the dissociation rate are much smaller than the parallel plasma density spreading rate. Thus, the plasma continuously spreads out in the parallel direction and does not accumulate along the fueling path. The high density injection leads to the sharp increase in the plasma density, termination of the inward molecular propagation and the molecular penetration depth starts to decrease.

Simulations with the HESEL model do not demonstrate the decrease in the penetration depth with increasing of the molecular beam density. This might relate to the fast plasma transport due to turbulence and poloidal plasma rotation, which spreads the density source more quickly compared to the steady trans-neut simulations. On the other hand, the self-blocking effect of SMBI might appear if it would be possible to overcome limitations of fixed boundary conditions in the HESEL model and increase the molecular beam density in our simulations. However, it should be emphasized that the critical density defined in the simulations with the trans-neut module of the $\mathrm{BOUT}++$ code is lower, than the experimental values, but at the same time the plasma density in the simulations increased in several orders

TABLE II. Approximation coefficients for an ionization efficiency as a function of a beam velocity shown in Fig. 11. $y$ is an equivalent of $\eta$.

\begin{tabular}{lccc}
\hline \hline & $a$ & $b$ & $y$ \\
\hline $\mathrm{q}=3$ & 7.74 & 0.28 & $y=a \cdot\left(V_{\text {smbi }}\right)^{b}$ \\
$\mathrm{q}=5.5$ small & 3.14 & 0.12 & $y=a \cdot\left(V_{\text {smbi }}\right)^{b}$ \\
$\mathrm{q}=5.5$ large & 0.36 & 0.58 & $y=a \cdot\left(V_{\text {smbi }}\right)^{b}$ \\
$\mathrm{q}=7.5$ & 0.094 & 0.66 & $y=a \cdot\left(V_{\text {smbi }}\right)^{b}$ \\
\hline
\end{tabular}

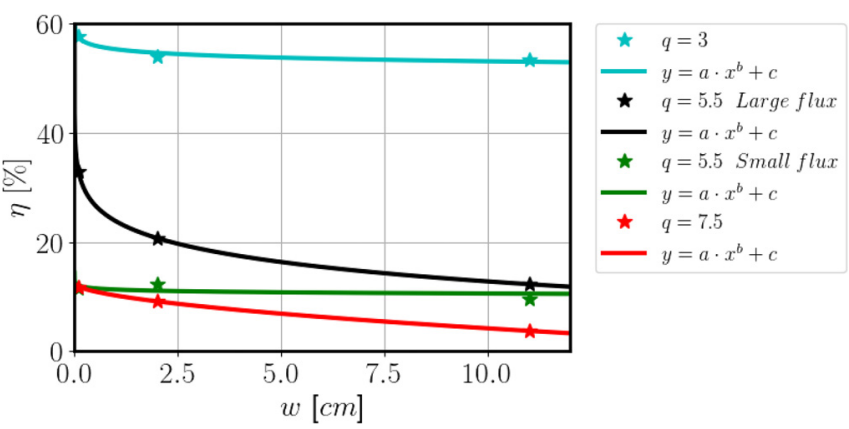

FIG. 12. Ionization efficiency as a function of a molecular beam width.

of magnitude. ${ }^{13}$ This observation indicates the importance of the correct implementation of the density source in the model and its spreading, especially in the parallel direction.

Another existing model for investigation of supersonic jets is a MHD code similar to the LLP pellet code. ${ }^{12}$ In this $1 D$ model, the jet expansion, deceleration of the ambient electrons and ions by the jet, resulted self-consistent electric field, and cooling of the ambient plasma are taken into account. Similar to the trans-neut module this model does not take into account turbulent transport. It has been shown that the ionization degree and the beam penetration depth strongly depend on the jet density. The critical jet density was around $N_{m o l}=10^{24} \mathrm{~m}^{-3}$ when the plasma electron is able to reach the center of the jet and the beam is ionized in plasma quite fast barely reaching the LCFS. The increase of the beam density over the critical value allows deeper penetration of the beam because the electrons cannot reach the center of the beam, which remains cold, dense, and neutral. $^{12}$

Comparison of these two models and our simulations with the HESEL code demonstrates that the density of the molecular beam is an essential parameter in describing SMBI into fusion plasma and the critical density values are very sensitive to the chosen physics model.

The LLP pellet code for supersonic jets and the trans-neut module have not taken into account the shape or width of the beam and have not provided any quantitative estimation of the fueling efficiency as a function of the beam parameters.

This article presents the first investigation of the influence of turbulence on SMBI. The study of the influence of plasma fluctuations on

TABLE III. Approximation coefficients for an ionization efficiency as a function of a beam width shown in Fig. 12. y is an equivalent of $\eta$.

\begin{tabular}{lcccc}
\hline & $a$ & $b$ & $c$ & $y$ \\
\hline $\mathrm{q}=3$ & $-1.5 \times 10^{4}$ & $6.3 \times 10^{-5}$ & $1.5 \times 10^{4}$ & $y=a \cdot\left(w_{\text {smbi }}\right)^{b}+c$ \\
$\mathrm{q}=5.5$ & -50.6 & 0.08 & 74.44 & $y=a \cdot\left(w_{\text {smbi }}\right)^{b}+c$ \\
large & & & & \\
$\mathrm{q}=5.5$ & $4.8 \times 10^{3}$ & $-6.5 \times 10^{-5}$ & $-4.84 \times 10^{3}$ & $y=a \cdot\left(w_{\text {smbi }}\right)^{b}+c$ \\
small & & & 12 & $y=a \cdot\left(w_{\text {smbi }}\right)^{b}+c$ \\
$\mathrm{q}=7.5$ & -2.2 & 0.57 & 12 & \\
\hline
\end{tabular}




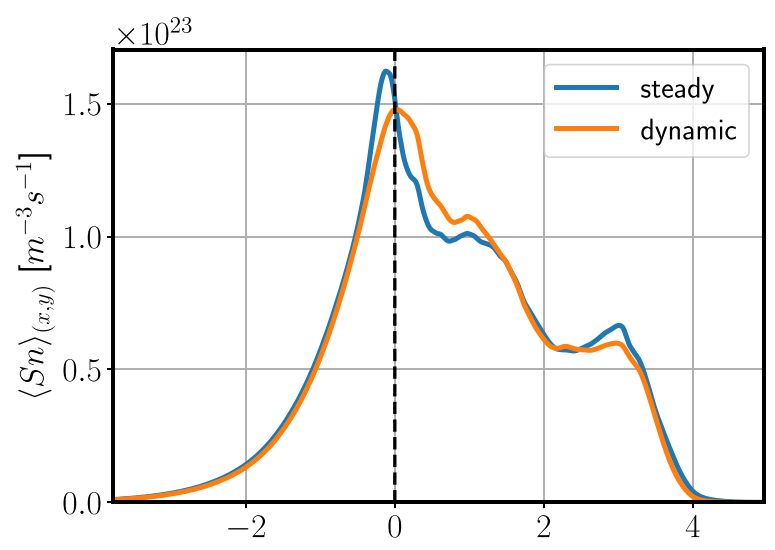

FIG. 13. Comparison of the density source profiles obtained in the simulations with full plasma dynamics and turbulence (dynamic) and the mean-field approximation (steady), where the dynamic plasma profiles are replaced by the time averaged fields. Simulations are performed with $q=5.5$ and the molecular beam parameters $w=0.1 \mathrm{~cm},\left\langle N_{\text {mol }}\right\rangle_{y}=7.4 \times 10^{22} \mathrm{~m}^{-3}, V=1000 \mathrm{~m} / \mathrm{s}$.

neutrals governed by diffusion in the SOL region has been performed in several works ${ }^{21,24,25,39}$ and the importance of the inclusion of the turbulent plasma transport in analysis of the neutral particle penetration through the SOL was mentioned. The supersonic molecular beam has some essential differences from the thermal neutrals described by diffusion equations. First, it is a high direct velocity, which makes the mean free path of injected molecules much longer. The smaller effect of turbulence on neutrals with a long mean free path is expected according to results in Refs. 25 and 21. Another essential difference is a narrow localization of the beam in poloidal direction, therefore only filaments on the beam penetration front affect the supersonic molecular beam. This is in analogy with the comparison of a 1D dynamical model with a 2D as was performed in Ref. 39, where the smaller discrepancy between the dynamical model and the mean-field approximation was observed in $2 \mathrm{D}$ simulations compared to $1 \mathrm{D}$. It was explained by the smaller size of blobs in $2 \mathrm{D}$ simulations compared to the size of the domain. Our results demonstrate the slight effect of plasma fluctuations on SMBI, that is, significantly different compared

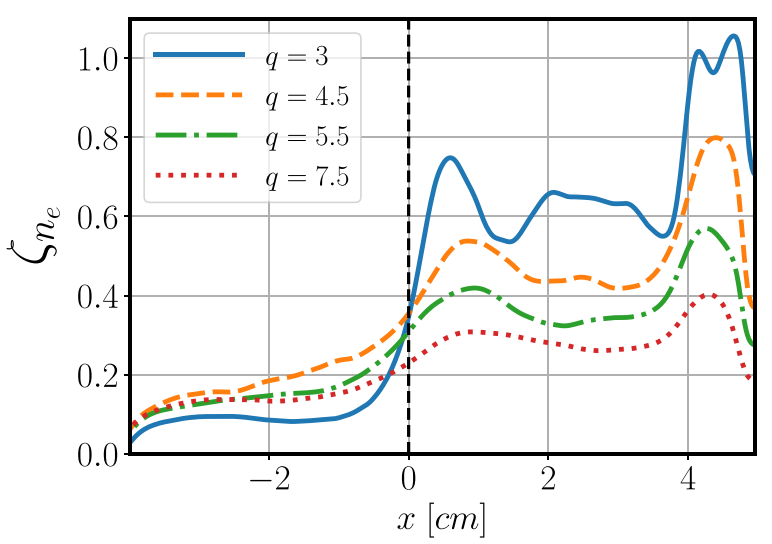

FIG. 14. Fluctuation level for the plasma density corresponding to simulations with different safety factors and without neutral injection.

to previous study of neutrals, but this finding might have reasonable explanations.

The decrease in the fluctuation level caused by SMBI is similar to the observations in Ref. 24, where the neutral gas injection reduced the fluctuation level of plasma density due to increase in the average plasma density. The quench of turbulence after SMBI is reported in Ref. 40 where simulations of L-H transitions in SMBI fueled plasmas are performed by using a meso-scale model.

\section{CONCLUSION}

A numerical investigation of the fueling efficiency in turbulent edge plasma as a function of the molecular beam parameters is performed. The self-consistent simulations of plasma-neutral interactions by the drift-fluid HESEL model combined with density continuity equations for molecules and atoms illuminate the influence of the beam parameters on the neutral penetration. A threshold molecular density was found in the simulations. This threshold is defined as the density source provided by the neutrals in the edge region comparable to the plasma source in the core and comparable to the particle losses in the SOL. Fueling efficiency grows with increasing molecular beam density above the threshold level while for the low-density beam
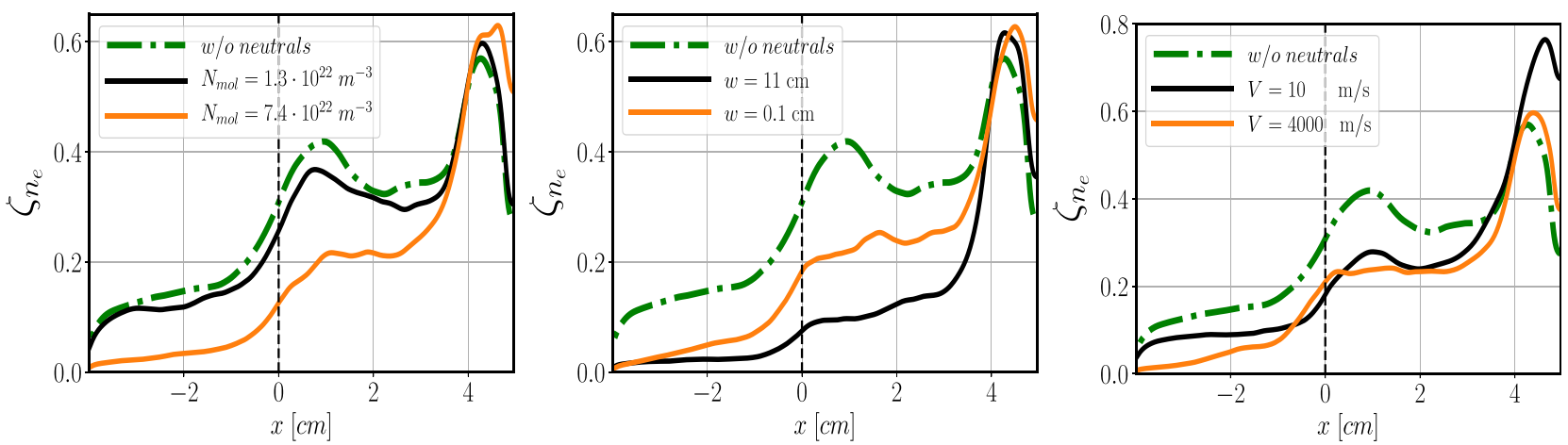

FIG. 15. Demonstration of the impact of neutral injection on the plasma fluctuations by comparison of the fluctuation level for the plasma density fluctuations without SMBI and after neutral injection. From left to right: effect of the molecular beam density $(V=1000 \mathrm{~m} / \mathrm{s}, \mathrm{W}=2 \mathrm{~cm})$, effect of the beam width $\left(\left\langle N_{\text {mol }}\right\rangle_{y}=7.4\right.$ $\left.\times 10^{22} \mathrm{~m}^{-3}, V=1000 \mathrm{~m} / \mathrm{s}\right)$, and effect of the beam velocity $\left(\left\langle N_{\mathrm{mol}}\right\rangle_{y}=1.3 \times 10^{22} \mathrm{~m}^{-3}, w=2 \mathrm{~cm}\right)$ 
injection the efficiency is almost independent of the molecular beam density. This is explained by the decrease in the plasma temperature in the SOL region and a reduction of the reaction rate coefficients. More than $90 \%$ of the density source in the edge region is produced by the ionization of Franck-Condon atoms and the rest is produced by ionization of molecules. The contribution of the molecular ionization to the density source grows with an increase in the molecular beam intensity. Independent of the molecular beam intensity, the increase in the injection velocity leads to the deeper penetration of neutrals and enhancement of the fueling efficiency. However, we observe a weaker growth rate of the ionization efficiency on the injection velocity with the increase in the plasma pressure or decrease in the injection flux.

The model used in this article allows for the investigation of SMBI into turbulent fusion plasmas. This model has some limitations, with its $2 \mathrm{D}$ nature as one of the most important. Simplified magnetic configuration without divertor region, neglecting of recycling effects, fixed inner boundary conditions and parametrization in parallel direction might affect the obtained results. An extension of the model to $3 \mathrm{D}$ and the inclusion of a realistic core-edge coupling and plasma-wall interactions will allow us for detailed investigations of the plasma fueling and finding of optimal injection parameters in different experimental scenarios.

Tokamaks of ITER size and parameters face the problem that particles will already be un-ionized in the SOL. We here try to estimate how SMBI might play out as an intermediate step between gas puff and pellet injection in helping to achieve ionization profiles which have significant reach into the confinement region.

\section{ACKNOWLEDGMENTS}

This work has been carried out within the framework of the EUROfusion Consortium and has received funding from the Euratom research and training programme 2014-2018 and 2019-2020 under Grant Agreement No. 633053. The views and opinions expressed herein do not necessarily reflect those of the European Commission.

\section{DATA AVAILABILITY}

The data that support the findings of this study are available from the corresponding author upon reasonable request.

\section{REFERENCES}

'J. W. Juhn, S. H. Hahn, S. H. Hong, J. I. Song, Y. O. Kim, K. P. Kim, J. S. Kim, and KSTAR Team, "Particle balance study for the fueling efficiency in KSTAR experiments 2013,” J. Korean Phys. Soc. 65, 1304-1311 (2014).

${ }^{2}$ D. L. Yu, C. Y. Chen, L. H. Yao, J. Q. Dong, B. B. Feng, Y. Zhou, Z. B. Shi, J. Zhou, X. Y. Han, W. L. Zhong, C. H. Cui, Y. Huang, Z. Cao, Y. Liu, L. W. Yan, Q. W. Yang, X. R. Duan, and Y. Liu, "Study of the high fuelling efficiency features of supersonic molecular beam injection," Nucl. Fusion 52, 082001 (2012).

${ }^{3}$ L. Yao, Supersonic Molecular Beam Injection in Fusion Plasma (Nova Science Publishers, Inc., 2006), p. 61.

${ }^{4}$ B. Pégourié, E. Tsitrone, R. Dejarnac, J. Bucalossi, G. Martin, J. Gunn, D. Frigione, D. Reiter, P. Ghendrih, and C. Clément, "Supersonic gas injection on Tore Supra,” J. Nucl. Mater. 313-316, 539-542 (2003).

${ }^{5}$ A. Murakami, J. Miyazawa, C. Suzuki, I. Yamada, T. Morisaki, R. Sakamoto, H. Yamada, and LHD Experiment Group, "Fueling characteristics of supersonic gas puffing applied to large high-temperature plasmas in the large helical device," Plasma Phys. Controlled Fusion 54, 055006 (2012).
${ }^{6}$ L. Yao, D. Zhao, B. Feng, C. Chen, Y. Zhou, X. Han, Y. Li, J. Bucalossi, and X. Duan, "Comparison of supersonic molecular beam injection from both low field side and high field side of HL-2A," Plasma Sci. Technol. 12, 529 (2010).

${ }^{7}$ L. Nie, J. Cheng, Y. Huang, L. W. Yan, L. H. Yao, B. B. Feng, J. Q. Dong, M. Xu, K. J. Zhao, D. L. Yu, W. L. Zhong, J. M. Gao, C. C. Yuan, Z. H. Huang, D. F. Kong, Z. Feng, C. H. Liu, K. Yao, J. Shang, Q. W. Yang, X. T. Ding, and X. R. Duan, "Intermittent convective transport suppressed by supersonic molecular beam injection on the HL-2A tokamak," Plasma Phys. Controlled Fusion 56, 055006 (2014).

${ }^{8}$ X. Zheng, J. Li, J. Hu, J. Li, R. Ding, B. Cao, and J. Wu, "Comparison between gas puffing and supersonic molecular beam injection in plasma density feedback experiments in EAST," Plasma Phys. Controlled Fusion 55, 115010 (2013).

${ }^{9}$ P. T. Lang, J. Neuhauser, J. Bucalossi, A. Chankin, D. P. Coster, R. Drube, R. Dux, G. Haas, L. D. Horton, S. Kalvin, G. Kocsis, M. Maraschek, V. Mertens, V. Rohde, V. Rozhansky, R. Schneider, I. Senichenkov, I. Veselova, and E. Wolfrum, "Impact of a pulsed supersonic deuterium gas jet on the ELM behaviour in ASDEX upgrade," Plasma Phys. Controlled Fusion 47, 1495-1516 (2005).

${ }^{10}$ X. Gao, Y. Jie, C. Xia, M. Wei, Y. Yang, S. Zhang, J. Zhao, L. Hu, Y. Zhu, J. Luo, Y. Zhao, N. Qiu, J. Li, B. Wan, G. Kuang, X. Zhang, X. Liu, X. Gong, Y. Bao, B. Lin, Z. Wu, Y. Li, Y. Shi, M. Song, P. Fu, X. Zhang, M. Zeng, A. Xie, N. Cui, H. Ruan, L. Wang, B. Sheng, S. Liu, W. Ye, K. Yang, J. Liu, Y. Cheng, H. Fan, S. Liu, X. Tong, J. Mao, X. Gu, J. Xie, and Y. Wan, "High density operation on the HT-7 superconducting tokamak,” Nucl. Fusion 40, 1875-1883 (2000).

${ }^{11}$ T. Mizuuchi, S. Kobayashi, M. Takeuchi, K. Mukai, N. Nishino, Y. Nakashima, S. Yamamoto, H. Okada, K. Nagasaki, T. Minami, S. Oshima, S. Kishi, H. Lee, K. Minami, Y. Takabatake, Y. Nakamura, K. Hanatani, S. Konoshima, and F. Sano, "Comparison between supersonic molecular-beam injection and conventional gas-puffing for plasma performance in heliotron J," in Proceedings of the 19th International Conference on Plasma-Surface Interactions in Controlled Fusion [J. Nucl. Mater. 415, S443-S446 (2011)].

${ }^{12}$ V. Rozhansky, I. Senichenkov, I. Veselova, D. Morozov, and R. Schneider, "Penetration of supersonic gas jets into a tokamak," Nucl. Fusion 46, 367-382 (2006).

${ }^{13}$ Z. H. Wang, X. Q. Xu, T. Y. Xia, and T. D. Rognlien, "2D simulations of transport dynamics during tokamak fuelling by supersonic molecular beam injection," Nucl. Fusion 54, 043019 (2014).

${ }^{14}$ Y. L. Zhou, Z. H. Wang, M. Xu, Q. Wang, L. Nie, H. Feng, and W. G. Sun, "Investigation of molecular penetration depth variation with SMBI fluxes," Chin. Phys. B 25, 095201 (2016).

${ }^{15}$ Y. F. Shi, Z. H. Wang, Q. L. Ren, A. P. Sun, D. L. Yu, W. F. Guo, and M. Xu, "Simulations of fast component and slow component of SMBI on HL-2A tokamak," Chin. Phys. B 26, 055201 (2017).

${ }^{16}$ S. J. Zweben, R. J. Maqueda, D. P. Stotler, A. Keesee, J. Boedo, C. Bush, S. Kaye, B. LeBlanc, J. L. Lowrance, V. J. Mastrocola, R. Maingi, N. Nishino, G. Renda, D. Swain, J. Wilgen, and NSTX Team, "High-speed imaging of edge turbulence in NSTX," Nucl. Fusion 44, 134-153 (2004).

${ }^{17}$ N. Yan, A. H. Nielsen, G. S. Xu, V. Naulin, J. J. Rasmussen, J. Madsen, H. Q. Wang, S. C. Liu, W. Zhang, L. Wang, and B. N. Wan, "Statistical characterization of turbulence in the boundary plasma of EAST," Plasma Phys. Controlled Fusion 55, 115007 (2013).

${ }^{18}$ F. Militello, P. Tamain, W. Fundamenski, A. Kirk, V. Naulin, and A. H. Nielsen, "Experimental and numerical characterization of the turbulence in the scrape-off layer of MAST," Plasma Phys. Controlled Fusion 55, 025005 (2013).

${ }^{19}$ O. E. Garcia, R. A. Pitts, J. Horacek, A. H. Nielsen, W. Fundamenski, J. P. Graves, V. Naulin, and J. Juul Rasmussen, "Turbulent transport in the TCV SOL," J. Nucl. Mater. 363-365, 575-580 (2007).

${ }^{20}$ D. A. D’Ippolito, J. R. Myra, and S. J. Zweben, "Convective transport by intermittent blob-filaments: Comparison of theory and experiment," Phys. Plasmas 18, 060501 (2011).

${ }^{21}$ A. Mekkaoui, Y. Marandet, D. Reiter, P. Genesio, J. Rosato, H. Capes, F. Catoire, L. Godbert-Mouret, M. Koubiti, and R. Stamm, "Transport of neutrals in turbulent scrape-off-layer plasmas,” J. Nucl. Mater. 415, S609 (2011).

${ }^{22}$ C. Wersal and P. Ricci, "Impact of neutral density fluctuations on gas puff imaging diagnostics,” Nucl. Fusion 57, 116018 (2017). 
${ }^{23}$ A. S. Thrysøe, M. Løiten, J. Madsen, V. Naulin, A. H. Nielsen, and J. J. Rasmussen, "Plasma particle sources due to interactions with neutrals in a turbulent scrape-off layer of a toroidally confined plasma," Phys. Plasmas 25, 032307 (2018).

${ }^{24}$ Y. Marandet, P. Tamain, R. Futtersack, P. Ghendrih, H. Bufferand, P. Genesio, and A. Mekkaoui, "Influence of neutral particles on scrape-off layer turbulence with application to the interpretation of fast camera data," in Proceedings of the 20th International Conference on Plasma-Surface Interactions in Controlled Fusion Devices [J. Nucl. Mater. 438, S518-S521 (2013)].

${ }^{25}$ D. Fan, Y. Marandet, P. Tamain, H. Bufferand, G. Ciraolo, P. Ghendrih, and E. Serre, "Effect of turbulent fluctuations on neutral particles transport with the TOKAM3X-EIRENE turbulence code," Nucl. Mater. Energy 18, 105-110 (2019).

${ }^{26}$ R. K. Janev, W. D. Langer, K. Evans, and D. E. Post, Elementary Processes in Hydrogen-Helium Plasmas (Springer-Verlag, Berlin, 1987).

${ }^{27}$ J. Madsen, V. Naulin, A. H. Nielsen, and J. J. Rasmussen, "Collisional transport across the magnetic field in drift-fluid models," Phys. Plasmas 23, 032306 (2016)

${ }^{28}$ A. H. Nielsen, J. J. Rasmussen, J. Madsen, G. S. Xu, V. Naulin, J. M. B. Olsen, M. Løiten, S. K. Hansen, N. Yan, L. Tophøj, and B. N. Wan, "Numerical simulations of blobs with ion dynamics," Plasma Phys. Controlled Fusion 59, 025012 (2016).

${ }^{29}$ F. L. Hinton and C. W. Horton, Jr., "Amplitude limitation of a collisional drift wave instability,” Phys. Fluids 14, 116 (1971).

${ }^{30}$ J. Olsen, A. H. Nielsen, J. J. Rasmussen, J. Madsen, T. Eich, B. Sieglin, and V. Naulin, "Scrape-off layer power fall-off length from turbulence simulations of ASDEX upgrade l-mode," Plasma Phys. Controlled Fusion 60, 085018 (2018).

${ }^{31}$ E. T. Meier and U. Shumlak, "A general nonlinear fluid model for reacting plasma-neutral mixtures,” Phys. Plasmas 19, 072508 (2012).
${ }^{32}$ D. Yu, C. Chen, L. Yao, B. Feng, X. Han, L. Yang, W. Zhong, Y. Zhou, K. Zhao, Y. Huang, Y. Liu, L. Yan, Q. Yang, J. Dong, and X. Duan, "Penetration characteristics of supersonic molecular beam injection on HL-2A tokamak," Nucl. Fusion 50, 035009 (2010).

${ }^{33}$ B. D. Dudson, A. Allen, G. Breyiannis, E. Brugger, J. Buchanan, L. Easy, S. Farley, I. Joseph, M. Kim, A. D. McGann et al., "BOUT: Recent and current developments," J. Plasma Phys. 81, 365810104 (2015).

${ }^{34}$ V. Naulin, J. Nycander, and J. J. Rasmussen, "Equipartition and transport in two-dimensional electrostatic turbulence," Phys. Rev. Lett. 81, 4148-4151 (1998).

${ }^{35} \mathrm{G}$. Avdeeva, "Investigations of high speed neutral particle injection into KSTAR plasmas," Ph.D. thesis (Department of Physics, Technical University of Denmark, 2019).

${ }^{36}$ Y. L. Zhou, Z. H. Wang, X. Q. Xu, H. D. Li, H. Feng, and W. G. Sun, "Comparisons between tokamak fueling of gas puffing and supersonic molecular beam injection in 2D simulations," Phys. Plasmas 22, 012503 (2015).

${ }^{37} \mathrm{X}$. Wu, H. Li, Z. Wang, H. Feng, and Y. Zhou, "Simulations of the effects of density and temperature profile on SMBI penetration depth based on the HL2A tokamak configuration,” Chin. Phys. B 26, 065201 (2017).

${ }^{38}$ Y. H. Wang, Z. H. Wang, W. Guo, Q. L. Ren, A. P. Sun, M. Xu, A. K. Wang, and N. Xiang, "Benchmark studies of BOUT ++ code and TPSMBI code on neutral transport during SMBI,” Phys. Lett. A 381, 1795-1806 (2017).

${ }^{39}$ A. S. Thrysøe, L. E. H. Tophøj, V. Naulin, J. J. Rasmussen, J. Madsen, and A. H. Nielsen, "The influence of blobs on neutral particles in the scrape-off layer," Plasma Phys. Controlled Fusion 58, 044010 (2016).

${ }^{40}$ K. Miki, P. H. Diamond, S. H. Hahn, W. W. Xiao, O. D. Gürcan, and G. R. Tynan, "Physics of stimulated L-H transitions," Phys. Rev. Lett. 110, 195002 (2013). 